\title{
Diffusion and subdiffusion of interacting particles on comb-like structures
}

\author{
O. Bénichou, ${ }^{1,2}$ P. Illien, ${ }^{1,2}$ G. Oshanin, ${ }^{1,2}$ A. Sarracino, ${ }^{1,2}$ and R. Voituriez ${ }^{1,2}$ \\ ${ }^{1}$ Sorbonne Universités, UPMC Univ Paris 06, UMR 7600, LPTMC, F-75005, Paris, France \\ ${ }^{2}$ CNRS, UMR 7600, Laboratoire de Physique Théorique de la Matière Condensée, F-75005, Paris, France
}

(Dated: May 29, 2022)

\begin{abstract}
We study the dynamics of a tracer particle (TP) on a comb lattice populated by randomly moving hard-core particles in the dense limit. We first consider the case where the TP is constrained to move on the backbone of the comb only, and, in the limit of high density of particles, we present exact analytical results for the cumulants of the TP position, showing a subdiffusive behavior $\sim t^{3 / 4}$. At longer times, a second regime is observed, where standard diffusion is recovered, with a surprising non analytical dependence of the diffusion coefficient on the particle density. When the TP is allowed to visit the teeth of the comb, based on a mean-field-like Continuous Time Random Walk description, we unveil a rich and complex scenario, with several successive subdiffusive regimes, resulting from the coupling between the inhomogeneous comb geometry and particle interactions. Remarkably, the presence of hard-core interactions speeds up the TP motion along the backbone of the structure in all regimes.
\end{abstract}

Subdiffusive motion of tracer particles in crowded media, e.g. biological cells, is widespread. Among the possible microscopic scenarios leading to this sublinear growth with time of the mean square displacement (MSD), the existence of geometric constraints related to the complexity of the environment plays an important role $[1,2]$. In this context, the comb model (see Fig. 1), in which a single particle moves on a two-dimensional space with the constraint that steps in the $x$ direction are only allowed when the $y$ coordinate of the particle positions is zero, has attracted considerable attention because of its simplicity and ability to reproduce subdiffusive behaviors of disordered systems [3].

Comb-like structures have indeed been introduced as a first step to model diffusion in more complicated fractal structures like percolation clusters, the backbone and teeth of the comb representing the quasilinear structure and dangling ends of percolation clusters [4]. The particle can spend a long time exploring a tooth, which results in a subdiffusive motion along the backbone with $\left\langle x^{2}(t)\right\rangle \propto t^{\alpha}$ with $\alpha=1 / 2$. Since, numerous results have been obtained for this model [5-12], including the determination of the occupation time statistics [13], of mean first-passage times between two nodes of a finite comb [14] or the case of fractional Brownian walks on comb-like structures [15].

In parallel, the comb model has been invoked to account for transport in real systems like spiny dendrites [10], diffusion of cold atoms [16] and mainly diffusion in crowded media like cells [17]. However, all existing studies have focused on single-particle diffusion, and interactions between particles have up to now been completely left aside. As an elementary model for diffusion of particles under short-range repulsive forces, we consider here excluded-volume interactions (EVIs) and focus on their impact on tracer dynamics on comb-like structures.

From a theoretical point of view, lattice systems of interacting particles represent a prototypical model in statistical physics that has generated a huge number of

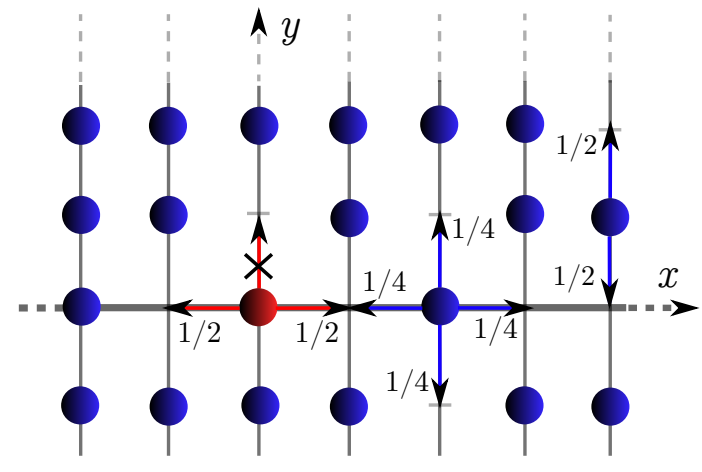

FIG. 1. Geometry of the system. The $x$-axis is designated as the backbone of the lattice, whereas the orthogonal lines are designated as the teeth. Jump rules of the particles in the case when the tracer particle (in red) is constrained to move on the backbone are given.

works both in the physical $[18,19]$ and mathematical literature [20]. The effect of EVIs in homogeneous lattices is well known [3]. In dimension $d \geq 2$, tracer diffusion has been shown to remain normal, with a non trivial diffusion coefficient resulting from many-body interactions and well approximated by the Nakazato-Kitahara approach [21]. In a "single-file" geometry, where particles cannot bypass each other, the impact of EVIs is stronger and results in a subdiffusive behavior $\left\langle x^{2}(t)\right\rangle \propto t^{\beta}$ with $\beta=1 / 2$ [22-27]. In this context, determining the effect of EVIs on systems with geometrical constraints appears an important question which does not seem to have received much attention. Notable exceptions are [28], where two particles only are involved, [29], which involves single-file motion with waiting times and thus does not consider explicitly geometric constraints, and [30], where tracer diffusion on DLA clusters was studied numerically and found to be not modified by the presence of EVIs. In this Letter, we show that, in contrast, EVIs deeply modify tracer diffusion on comb-like structures. Focusing on 
the high density limit, we show analytically that, due to a subtle interplay between the inhomogeneous comb geometry and crowding effects, the dynamics displays several regimes of anomalous diffusion. We find in particular that, surprisingly, the presence of EVIs can speed up tracer diffusion along the backbone of the structure.

Model.- We consider the 2-dimensional comb $\mathbf{C}_{2}$, which is a subgraph of $\mathbb{Z}^{2}$ obtained by removing all the edges parallel to the $x$-axis, except from the $x$-axis itself. This lattice is populated by $N$ hard-core particles with average density $\rho=N / V$, where $V$ is the number of sites. Each particle performs a symmetric nearestneighbor random walk, constrained by hard-core interactions. We add a tracer particle (TP) at the origin, performing a symmetric nearest-neighbor random walk, and focus on its dynamics in the dense limit, where the vacancy density $\rho_{0}=1-\rho \ll 1$. In this limit, it is more convenient to describe the vacancy dynamics instead of describing the dynamics of all the particles. We assume here that, at each time step, each vacancy exchanges its position with one of the neighboring particles, with jump probabilities that depend on the position on the lattice; see the Supplemental Material (SM) [31] for the explicit definition of those evolution rules.

Case of a TP restricted to the backbone- We first assume that the TP (and only the TP) is constrained to move on the backbone. This particular case is important for several reasons. (i) It mimics the case where the tracer is different from the bath particles, and is not able to visit the teeth of the comb. (ii) At the theoretical level, it appears as an extension of the famous single-file geometry in which, due do the possibility for the bath particles to visit the teeth, the particles can bypass each other. An interesting question is to know if the dynamics is still anomalous in this case, and if so, with which exponent; (iii) finally, as shown below, solving this auxiliary problem will allow us to determine the dynamics of the TP in the general case where the TP can access the teeth of the comb.

Let $X_{t}$ be the random variable characterizing the position of the TP along the backbone at time $t$. We aim at computing the cumulants of arbitrary order $n$ of this variable, denoted by $\kappa^{(n)}(t)$. These quantities are generated by the cumulant generating function $(\mathrm{CGF}) \Psi_{t}(k) \equiv$ $\ln \left\langle\mathrm{e}^{i k X_{t}}\right\rangle=\ln \left[\widetilde{P}_{t}(k)\right]$, where $\widetilde{P}_{t}(k)=\sum_{X} e^{i k X} P_{t}(X)$ is the Fourier transform of the probability $P_{t}(X)$ to find the TP at position $X$ at time $t$. Following the method developed in [32] and recently used to study driven diffusion in one-dimensional geometries [33], we first consider the case where there is a single vacancy on the lattice. Let $P_{t}^{(1)}(X \mid \boldsymbol{Z})$ be the probability to find the TP at position $X$ at time $t$ knowing that the vacancy started from site $\boldsymbol{Z}$. Summing over all the passages of the vacancy to the
TP location, one gets:

$$
\begin{aligned}
& P_{t}^{(1)}(X \mid \boldsymbol{Z})=\delta_{X, 0}\left(1-\sum_{j=0}^{t} F_{j}(\mathbf{0} \mid \boldsymbol{Z})\right) \\
+ & \sum_{p=1}^{+\infty} \sum_{m_{1}, \ldots, m_{p}=1}^{+\infty} \sum_{m_{p+1}=0}^{+\infty} \delta_{m_{1}+\ldots+m_{p+1}, t} \delta_{X, \frac{\operatorname{sgn}\left(\boldsymbol{Z} \cdot \mathbf{e}_{\mathbf{1}}\right)+(-1)^{p+1}}{2}} \\
\times & \left(1-\sum_{j=0}^{m_{p+1}} F_{j}\left(\mathbf{0} \mid(-1)^{p} \mathbf{e}_{\mathbf{1}}\right)\right) \\
\times & F_{m_{p}}\left(\mathbf{0} \mid(-1)^{p+1} \mathbf{e}_{\mathbf{1}}\right) \ldots F_{m_{1}}(\mathbf{0} \mid \boldsymbol{Z}),
\end{aligned}
$$

where $F_{t}(\mathbf{0} \mid \boldsymbol{Z})$ is the probability for the vacancy to reach the origin for the first time at time $t$, knowing that it started from site $\boldsymbol{Z}$, and $\mathbf{e}_{\mathbf{1}}$ stands for the unit vector in the $x$ direction. The first term in the right-hand side of Eq. (1) represents the event that at time $t$, the TP has not been visited by any vacancy, while the second one results from a partition both on the number $p$ of visits and waiting times $m_{i}$ between visits of the TP by the vacancy. Computing the generating function associated with this propagator $\widehat{p}_{ \pm 1}(X ; \xi) \equiv \widehat{P}^{(1)}\left(X \mid \pm \boldsymbol{e}_{1} ; \xi\right)$, where $\widehat{\phi}(\xi)$ denotes the discrete Laplace transform $\widehat{\phi}(\xi) \equiv \sum_{t=0}^{\infty} \phi_{t} \xi^{t}$, and noticing that for symmetry reasons $\widehat{F}\left(\mathbf{0} \mid e_{1} ; \xi\right)=$ $\widehat{F}\left(\mathbf{0} \mid-\boldsymbol{e}_{1} ; \xi\right) \equiv \widehat{F}_{1}$, one gets

$$
\widehat{p}_{ \pm 1}(X ; \xi)=\frac{\delta_{X, 0}\left(1-\widehat{F}_{1}\right)+\delta_{X, \pm 1} \widehat{F}_{1}\left(1-\widehat{F}_{1}\right)}{\left(1-\widehat{F}_{1}^{2}\right)(1-\xi)} .
$$

We then study the case where the concentration of vacancies on the lattice $\rho_{0}$ is finite but very small. For clarity, we first assume that the lattice has a finite number of sites $N$, and that it is populated by $M$ vacancies, so that $M=\rho_{0} N$. Consequently, the probability $P_{t}\left(X \mid\left\{\boldsymbol{Z}_{j}\right\}\right)$ to find the TP at position $X$ as a result of its interactions with $M$ vacancies initially located at sites $\boldsymbol{Z}_{1}, \cdots, \boldsymbol{Z}_{M}$, is given by

$$
P_{t}\left(X \mid\left\{\boldsymbol{Z}_{j}\right\}\right)=\sum_{\boldsymbol{Z}_{1}, \cdots, \boldsymbol{Z}_{M}} \delta_{X, Y_{1}+\cdots+Y_{M}} P_{t}\left(\left\{Y_{j}\right\} \mid\left\{\boldsymbol{Z}_{j}\right\}\right),
$$

where $P_{t}\left(\left\{Y_{j}\right\} \mid\left\{\boldsymbol{Z}_{j}\right\}\right)$ is the conditional probability that during the time interval $t$ the TP moved of a distance $Y_{j}$ due to its interactions with the $j$-th vacancy. To leading order in $\rho_{0}$, the vacancies contribute independently to the displacement of the TP, so that in Fourier variable, $\widetilde{P}_{t}^{(M)}(k)=\left[\widetilde{P}_{t}^{(1)}(k)\right]^{M}$, where $\widetilde{P}_{t}^{(j)}(k)$ is the Fourier transform of the probability distribution to find the TP at position $X$ at time $t$, knowing that there are $j$ vacancies on the lattice, and averaged over the initial position of the vacancies, which is assumed to be uniform. As shown below, the choice of the initial distribution of the vacancies may actually have a dramatic effect on the behavior of the TP.

Finally, in the thermodynamic limit in which $N, M \rightarrow$ $\infty$ with fixed $\rho_{0}=M / N$, and using Eqs. (2) we get the 


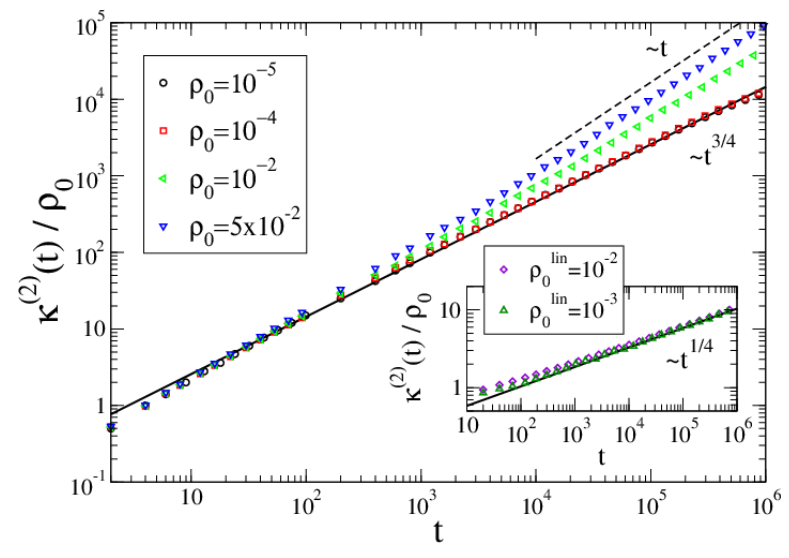

FIG. 2. (Color online) Variance rescaled by $\rho_{0}$ computed from Monte-Carlo simulations of vacancy dynamics. The full line represents the analytical result (5). Inset: case where all vacancies are initially placed on the backbone (the full line represents Eq. (12.))

Fourier Laplace transform of the CGF

$$
\widehat{\Psi}(k ; \xi) \underset{\rho_{0} \rightarrow 0}{\sim}-2 \rho_{0} \frac{H(\xi)}{(1-\xi)\left(1+\widehat{F}_{1}\right)}(1-\cos k),
$$

where we defined $H(\xi) \equiv \sum_{x=1}^{\infty} \sum_{y=-\infty}^{\infty} \widehat{F}(\mathbf{0} \mid x, y ; \xi)$, and where we used the symmetry relation $\sum_{\boldsymbol{Z} \neq 0} \widehat{F}^{*}\left(\mathbf{0}\left|\boldsymbol{e}_{1}\right| \boldsymbol{Z}\right)=\sum_{\boldsymbol{Z} \neq 0} \widehat{F}^{*}\left(\mathbf{0}\left|-\boldsymbol{e}_{1}\right| \boldsymbol{Z}\right)$. Consequently, the determination of the CGF amounts to the calculation of the quantity $H(\xi)$ and the first passage density $\widehat{F}_{1}$, which are given in the SM [31].

Expanding $\widehat{\Psi}(k ; \xi)$ in powers of $k$ from Eq. (4), focusing on the large time limit $\xi \rightarrow 1^{-}$and using a Tauberian theorem [34] we then get the following exact expression for the cumulants (see SM [31] for details)

$$
\lim _{\rho_{0} \rightarrow 0} \frac{\kappa^{(2 n)}(t)}{\rho_{0}} \underset{t \rightarrow \infty}{\sim} \frac{1}{2^{5 / 4} \Gamma(7 / 4)} t^{3 / 4}
$$

Several comments are in order. (i) First, one notices that all cumulants of the same parity are equal. This indicates that the probability distribution $P_{t}(X)$ is a Skellam distribution [35], originally defined as the p.d.f. associated with the difference of two Poissonian random variables. In particular, the rescaled position $X_{t} / t^{3 / 8}$ is asymptotically normally distributed. (ii) The exponent $3 / 4$ is intermediate between a "normal" diffusion exponent, and the single-file diffusion exponent $1 / 2$. (iii) While the analytical predictions of the cumulants are successfully compared to results obtained from Monte Carlo simulations at intermediate times (see Fig. 2), at long times a crossover towards a standard diffusive behavior is observed.

We present below a theoretical argument that accounts for this intriguing behavior. The key point underlying this crossover is that the above analytical results are derived by taking the limit $\rho_{0} \rightarrow 0$ before the long time limit. In this case, to leading order, the TP does not move before being reached by a given vacancy. In fact, the TP actually diffuses due to its interactions with the other vacancies. Because of this effective diffusion of the TP, each vacancy experiences an additional symmetric jump probability in the $x$ direction, denoted by $D\left(\rho_{0}\right)$, even when they are on the teeth. Thus, in the reference frame of the $\mathrm{TP}$, the vacancies are allowed to jump from one tooth to another (with a probability that vanishes as $\rho_{0} \rightarrow 0$ ) and their motion is now effectively two-dimensional. Qualitatively, we are facing a two-dimensional situation, and regular diffusion is thus expected at large times (see introduction). Quantitatively, the approach developed previously can be extended to calculate the variance of $X_{t}$, in this case where the vacancies perform $2 \mathrm{D}$ random walks, but can reach the TP only from the backbone (see SM [31]), yielding

$$
\widehat{\kappa}^{(2)}(\xi)=-2 \rho_{0} \frac{\Sigma\left(\xi, \rho_{0}\right)\left(\widehat{F}_{1}^{*}-\widehat{F}_{-1}^{*}-1\right)}{\left(\widehat{F}_{1}^{*}-1+\widehat{F}_{-1}^{*}\right)\left(\widehat{F}_{1}^{*}+1-\widehat{F}_{-1}^{*}\right)},
$$

where we defined $\widehat{F}_{ \pm 1}^{*} \equiv \widehat{F}^{*}\left(\mathbf{0}\left|\boldsymbol{e}_{1}\right| \boldsymbol{e}_{ \pm 1} ; \xi, \rho_{0}\right)$ and $\Sigma\left(\xi, \rho_{0}\right) \equiv \sum_{\boldsymbol{Z} \neq \mathbf{0}} \widehat{F}^{*}\left(\mathbf{0}\left|\boldsymbol{e}_{1}\right| \boldsymbol{Z} ; \xi, \rho_{0}\right)$, with $F_{t}^{*}\left(\mathbf{0}\left|\boldsymbol{e}_{1}\right| \boldsymbol{Z} ; \rho_{0}\right)$ the probability for a vacancy to reach the origin for the first time at time $t$ knowing that it was at site $\boldsymbol{e}_{1}$ at time $t-1$ and that it started from site $\boldsymbol{Z}$. Relying on renewaltype equations, the conditional first-passage time densities $\widehat{F}^{*}$ are related to the propagators of the vacancies random walk, which are themselves evaluated with an extension of the method presented in [36] to treat diffusion on inhomogeneous lattices (see SM [31]). It is finally found that

$$
\begin{aligned}
\widehat{F}_{-1}^{*} & \simeq \frac{\sqrt{2}}{\pi} \sqrt{D\left(\rho_{0}\right)} \ln \frac{1}{D\left(\rho_{0}\right)}-\frac{\pi \sqrt{D\left(\rho_{0}\right)}}{\ln \frac{1}{1-\xi}}+\ldots \\
\widehat{F}_{1}^{*} & \simeq 1-\frac{\pi \sqrt{D\left(\rho_{0}\right)}}{\ln \frac{1}{1-\xi}}+\ldots
\end{aligned}
$$

where the symbol $\simeq$ stands for the long-time limit $(\xi \rightarrow$ 1 ) for a fixed value of $\rho_{0}$. As expected, $\widehat{F}_{-1}^{*} \underset{\rho_{0} \rightarrow 0}{\rightarrow} 0$. Next, $\Sigma\left(\xi, \rho_{0}\right)$ is conveniently estimated in SM [31] by relying on the continuous-space description given in [5]:

$$
\Sigma\left(\xi, \rho_{0}\right) \underset{\xi \rightarrow 1}{\propto} \frac{\sqrt{D\left(\rho_{0}\right)}}{(1-\xi) \ln \frac{1}{1-\xi}} .
$$

Plugging Eqs. (7), (8) and (9) into (6), and using a Tauberian theorem, we finally obtain

$$
\lim _{t \rightarrow \infty} \frac{\kappa^{(2)}(t)}{t} \underset{\rho_{0} \rightarrow 0}{\propto} \rho_{0} \sqrt{D\left(\rho_{0}\right)} \ln \frac{1}{D\left(\rho_{0}\right)} .
$$

This equation defines the effective diffusion coefficient $D\left(\rho_{0}\right)=\lim _{t \rightarrow \infty}\left(\kappa^{(2)}(t) / 2 t\right)$ self-consistently when $\rho_{0} \rightarrow$ 0 , and finally yields the following expression of the variance in the ultimate regime:

$$
\lim _{t \rightarrow \infty} \frac{\kappa^{(2)}(t)}{t} \underset{\rho_{0} \rightarrow 0}{\propto} \rho_{0}^{2}\left(\ln \frac{1}{\rho_{0}}\right)^{2} .
$$


These results thus show that the limits $\rho_{0} \rightarrow 0$ and $t \rightarrow \infty$ do not commute leading to an ultimate diffusive behavior [37]. However, due to a subtle coupling between EVIs and the geometrical constraints involved in the comb geometry, this diffusive regime displays a non analytical dependence on the vacancy density, checked numerically in Fig. 4 of the SM [31]. This is markedly different from the case of homogeneous lattices where a linear behavior with $\rho_{0}$ is found [32]. In addition, the comparison between Eqs. (5) and (11) shows that the crossover time between the two regimes behaves like $t_{\times} \sim\left(\rho_{0} \ln \left(\rho_{0}\right)\right)^{-4}$, which can be very large for dense systems. As a result, the subdiffusive behavior of the first regime is long-lived and potentially observable in real systems. We now consider several extensions of these results.

Influence of the initial conditions. The previous results were obtained assuming that the vacancies were initially uniformly distributed on the lattice. We now assume that they are initially located only on the backbone, with a linear density $\rho_{0}^{\text {lin }}$ defined as the number of vacancies divided by the length of the backbone. Averaging over this initial distribution, which actually amounts to restricting the sum over the initial points to the only backbone in Eq. (4), it is found that (see SM [31])

$$
\kappa^{(2 n)}(t) \underset{\rho_{0}^{\mathrm{lin}} \rightarrow 0}{\sim} \frac{\rho_{0}^{\operatorname{lin}}}{2^{7 / 4} \Gamma(5 / 4)} t^{1 / 4} .
$$

Consequently, the time dependence of the cumulants is modified in a dramatic way: the cumulants now grow as $t^{1 / 4}$. This analytical prediction is successfully confronted to numerical simulations (see inset of Fig. 2). This spectacular slowdown of the dynamics with respect to the uniform initial conditions is compatible with Eq. (5), where now $\rho_{0}$ strictly vanishes. Interestingly, in this case, $t_{\times} \rightarrow \infty$, so that there is no cross-over to a diffusive regime.

d-dimensional comb.- The previous results can also be generalized to the important case of a $d$-dimensional comb $\mathbf{C}_{d}$, widely studied in the literature [3, 38, 39], which is defined recursively: starting from $\mathbf{C}_{1}$ (a onedimensional lattice), $\mathbf{C}_{d}$ is obtained from $\mathbf{C}_{d-1}$ by attaching at each point a two-way infinite path (see figure in SM [31]). It is found that for uniform initial conditions, the even cumulants all behave like

$$
\lim _{\rho_{0} \rightarrow 0} \frac{\kappa^{(2 n)}(t)}{\rho_{0}} \underset{t \rightarrow \infty}{\propto} t^{1-\frac{1}{2^{d}}},
$$

and eventually cross-over to a diffusive linear in time regime for $d \geq 2$. Note that in the case of the $d=1$ comb, single-file subdiffusion $\kappa^{(2 n)}(t) \sim \sqrt{t}$ is recovered.

Finally, reminding that single-file diffusion has been shown to be a realization of a fractional Brownian motion with Hurst exponent 1/4 [40], we conjecture that tracer diffusion in a crowded $d$-comb is more generally a realization of a fractional Brownian motion of Hurst exponents $H=\left(2^{d}-1\right) / 2^{d+1}$.

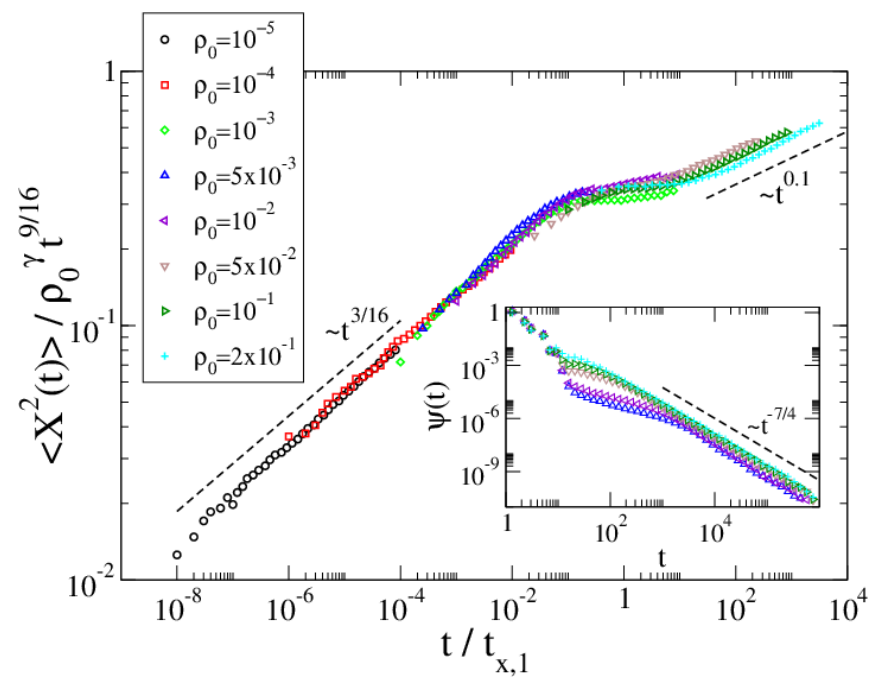

FIG. 3. Variance of the TP visiting the teeth, measured for different values of $\rho_{0}$. The collapse of the curves is obtained by rescaling time by the crossover time $t_{\times, 1}=1 / \rho_{0}^{2}$, predicted by our analytical approach, and the variance by $\rho_{0}^{\gamma}$ with $\gamma \simeq 0.62$, numerically obtained. The plateau at intermediate times confirms the prediction of Eq. (15). For $\rho_{0} \leq 10^{-3}$ the reported results are obtained via numerical simulations of vacancy dynamics, while for $\rho_{0}>10^{-3}$ via particle dynamics (see SM [31]).

Case of a TP visiting the teeth- We finally come back to the original problem of a tracer on a crowded 2-comb, where the TP is identical to the bath particles and thus allowed to visit the teeth of the comb. The displacement of the TP along the backbone can be analyzed in a meanfield description that decouples the motion of the TP in a tooth from the dynamics of other bath particles as a Continuous Time Random Walk, whose waiting time distribution $\psi(t)$ describes the time the TP spends on a given tooth of the crowded comb. Noting that the motion of the TP along a tooth is close to a single-file motion, we expect that the transverse MSD behaves like $\left\langle y^{2}(t)\right\rangle \propto \sqrt{\rho_{0}^{2} t}[24]$, in the dense limit. In turn, this leads to two different regimes for $\psi(t)$ : for $t \ll t_{\times, 1} \equiv 1 / \rho_{0}^{2}$, $\left\langle y^{2}(t)\right\rangle \ll 1$, the TP has not had time to explore a tooth because of the other crowding bath particles of the tooth, and the mean time spent on the tooth is finite; for $t \gg$ $t_{\times, 1}, \psi(t) \propto 1 / t^{\mu}$, with $\mu=7 / 4$, as obtained in $[41,42]$ and checked numerically (see inset of Fig 3 ).

The MSD $\left\langle X^{2}\right\rangle$ of the TP along the backbone is then related to the MSD $\kappa^{(2)}$ of the TP restricted to the backbone by using the standard Montroll Weiss relation [34]:

$$
\widehat{\left\langle X^{2}\right\rangle}(\xi)=\frac{1-\widehat{\psi}(\xi)}{1-\xi} \widehat{\kappa^{(2)}}(\widehat{\psi}(\xi))
$$

Combining the two temporal regimes of $\kappa^{(2)}(t)$ determined previously with the two regimes of $\psi(t)$, we finally 
obtain that $\left\langle X^{2}(t)\right\rangle$ displays three non trivial regimes :

$$
\left\langle X^{2}(t)\right\rangle \propto \begin{cases}t^{3 / 4} & \text { if } t \ll t_{\times, 1}, \\ t^{3 / 4(\mu-1)}=t^{9 / 16} & \text { if } t \ll, 1 \ll t \ll t_{\times, 2}, \\ t^{\mu-1}=t^{3 / 4} & \text { if } t \gg t_{\times, 2}\end{cases}
$$

where $t_{\times, 2}$ is a second crossover time (whose explicit dependency on $\rho_{0}$ is not provided by our approach). The comparison with numerical simulations shown in Fig. 3 reveals that: (i) three temporal regimes with expected crossover time $t_{\times, 1}$ are indeed observed; (ii) the exponents of the two first are in good agreement with our analytical prediction (15); (iii) the increase of $\left\langle X^{2}(t)\right\rangle$ observed in the last regime is in qualitative agreement with (15) but the quantitative determination of the corresponding exponent would require more extensive simulations. Remarkably, it is found that in all regimes, the dynamics of the TP along the backbone is faster than in the absence of bath particles, where $\left\langle X^{2}(t)\right\rangle \sim t^{1 / 2}$. In other words, the motion of the TP is accelerated along the backbone by EVIs. This surprising behavior results from two competing effects quantified by our approach: hard-core interactions hinder the motion of the TP along the backbone but in the same time reduce the time lost by the TP in the teeth.
[1] R. Metzler and J. Klafter, Phys. Rep. 339, 1 (2000).

[2] S. Condamin, V. Tejedor, R. Voituriez, O. Bénichou, and J. Klafter, Proc. Nat. Acad. Sci. 105, 5675 (2008).

[3] D. Ben-Avraham and S. Havlin, Diffusion and reactions in fractals and disordered systems (Cambridge University Press, 2005).

[4] G. Weiss and S. Havlin, Physica A 134, 474 (1986).

[5] V. Arkhincheev and E. Baskin, Zh. Exper. Teor. Fiziki 300, 161 (1991).

[6] R. Burioni and D. Cassi, J. Phys. A: Math. Gen. 38, R45 (2005).

[7] H. Frauenrath, Prog. Polym. Sci. 30, 325 (2005).

[8] A. Iomin, Phys. Rev. E 83, 052106 (2011).

[9] D. Villamaina, A. Sarracino, G. Gradenigo, A. Puglisi, and A. Vulpiani, J. Stat. Mech. , L01002 (2011), 1101.4097.

[10] V. Méndez and A. Iomin, Chaos, Solitons \& Fractals 53, 46 (2013).

[11] E. K. Lenzi, L. R. da Silva, A. A. Tateishi, M. K. Lenzi, and H. V. Ribeiro, Phys. Rev. E 87, 012121 (2013).

[12] E. Agliari, A. Blumen, and D. Cassi, Phys. Rev. E 89, 1 (2014).

[13] A. Rebenshtok and E. Barkai, Phys. Rev. E 88, 052126 (2013).

[14] E. Agliari, F. Sartori, L. Cattivelli, and D. Cassi, Phys. Rev. E 91, 1 (2015).

[15] H. V. Ribeiro, A. A. Tateishi, L. G. A. Alves, R. S. Zola, and E. K. Lenzi, New J. Phys. 16, 093050 (2014).

[16] Y. Sagi, M. Brook, I. Almog, and N. Davidson, Phys. Rev. Lett. 108, 3 (2012).

[17] F. Höfling and T. Franosch, Rep. Prog. Phys. 76, 046602 (2013).

[18] T. Chou, K. Mallick, and R. K. P. Zia, Rep. Prog. Phys. 74, 116601 (2011).

[19] E. Levine, Y. Kafri, and D. Mukamel, Phys. Rev. E 64, 026105 (2001).

[20] H. Spohn, Large Scale Dynamics of Interacting Particles (Springer-Verlag, 1991).

[21] K. Nakazato and K. Kitahara, Prog. Theor. Phys. 64, 2261 (1980).

[22] T. Harris, J. Appl. Probab. 2, 323 (1965).

[23] D. G. Levitt, Phys. Rev. A 8, 3050 (1973).

[24] R. Arratia, Ann. Probab. 11, 362 (1983).

[25] N. Leibovich and E. Barkai, Phys. Rev. E 88, 032107
(2013).

[26] P. L. Krapivsky, K. Mallick, and T. Sadhu, Phys. Rev. Lett. 113, 078101 (2014).

[27] C. Hegde, S. Sabhapandit, and A. Dhar, Phys. Rev. Lett. 113, 120601 (2014).

[28] R. Burioni, D. Cassi, G. Giusiano, and S. Regina, J. Phys. A 35, 217 (2002).

[29] L. P. Sanders, M. A. Lomholt, L. Lizana, K. Fogelmark, R. Metzler, and T. Ambjörsson, New J. Phys. 16, 113050 (2014).

[30] C. Amitrano, A. Bunde, and H. Stanley, J. Phys. A 923 (1985).

[31] See Supplementa Material at ..., which includes Ref. [43, 44], for details on calculations and simulations.

[32] M. J. A. M. Brummelhuis and H. J. Hilhorst, Physica A 156, 575 (1989).

[33] P. Illien, O. Bénichou, C. Mejía-Monasterio, G. Oshanin, and R. Voituriez, Phys. Rev. Lett. 111, 38102 (2013).

[34] B. D. Hughes, Random Walks and Random Environments: Random walks, Volume 1 (Oxford University, New York, 1995).

[35] J. G. Skellam, J. Royal Stat. Soc. 109, 296 (1946).

[36] T. Nieuwenhuizen, S. Klumpp, and R. Lipowsky, Phys. Rev. E 69, 061911 (2004).

[37] A similar non-inversion mechanism was found in [45]. However, in contrast to what is found here, this effect exists only when the TP experiences a non zero bias.

[38] D. Cassi and S. Regina, Mod. Phys. Lett. B 6, 1397 (1992).

[39] D. Bertacchi and F. Zucca, J. Aust. Math. Soc. 75, 325 (2003).

[40] C. Landim and S. Volchan, Stoch. Proc. Appl. 85, 139 (2000).

[41] J. Krug, H. Kallabis, S. Majumdar, S. Cornell, A. Bray, and C. Sire, Phys. Rev. E 56, 2702 (1997).

[42] G. M. Molchan, Commun. Math. Phys. 205, 97 (1999).

[43] W. Woess, Random Walks on Infinite Graphs and Groups (Cambridge University Press, 2000).

[44] V. E. Arkhincheev, Physica A 307, 131 (2002).

[45] O. Bénichou, A. Bodrova, D. Chakraborty, P. Illien, A. Law, C. Mejía-Monasterio, G. Oshanin, and R. Voituriez, Phys. Rev. Lett. 111, 260601 (2013). 


\section{Supplemental Material}

\section{EVOLUTION RULES OF THE VACANCIES}

In the main text, we first studied the case where the tracer particle (TP) is constrained to move on the backbone of the comb. The evolution rules of the particles where given in Fig. 1: (i) the TP jumps on each of the neighboring sites of the backbone with probability $1 / 2$; (ii) if a bath particle is located on a tooth of the comb, it jumps on each of the neighboring sites with probability $1 / 2$; (iii) if a bath particle is located on the backbone of the structure, it jumps on each of the neighboring sites with probability $1 / 4$. The dynamics is constrained with hardcore interactions, which means that there is at most one particle per site.

In the high-density limit, there are very few vacancies on the lattice, and it is more convenient to describe their dynamics instead of describing the dynamics of all the particles. The events where two vacancies are on neighboring sites are of order $\mathcal{O}\left(\rho_{0}^{2}\right)$ (where $\rho_{0}$ is the density of vacancies on the lattice). As we focus on the results at leading order in $\rho_{0}$, we do not take these events into account. We adopt a discrete-time evolution, and assume that at each time step, each vacancy exchanges its position with one of the neighboring particles. Depending on the position $\boldsymbol{Z}$ of the vacancy (see Fig. 4), its jump probabilities are defined as follows:

- if the vacancy is on a tooth of the comb but not adjacent to the backbone, it exchanges its position with the same probability with each of the neighboring particles.

- if the vacancy is at $\boldsymbol{Z}$, adjacent to the backbone but not to the TP, the particle located at $\boldsymbol{Z}-\boldsymbol{e}_{2}$ (resp. $\boldsymbol{Z}+\boldsymbol{e}_{2}$ ) has a probability $1 / 4$ (resp. 1/2) to exchange its position with that of the vacancy. Consequently, the vacancy has a probability proportional to $1 / 4$ (resp. proportional to $1 / 2$ ) to jump in direction $-\boldsymbol{e}_{2}$ (resp. $\boldsymbol{e}_{2}$ ), so that

$$
\begin{aligned}
& p\left(\boldsymbol{Z}-\boldsymbol{e}_{2} \mid \boldsymbol{Z}\right)=\mathcal{Z} \times \frac{1}{4}, \\
& p\left(\boldsymbol{Z}+\boldsymbol{e}_{2} \mid \boldsymbol{Z}\right)=\mathcal{Z} \times \frac{1}{2},
\end{aligned}
$$

where $p\left(\boldsymbol{r} \mid \boldsymbol{r}^{\prime}\right)$ is the probability for a vacancy to jump from site $\boldsymbol{r}^{\prime}$ to site $\boldsymbol{r}$ in a single step, and where $\mathcal{Z}$ is a normalization constant. With the normalization condition $p\left(\boldsymbol{Z} \rightarrow \boldsymbol{Z}-\boldsymbol{e}_{2}\right)+p\left(\boldsymbol{Z} \rightarrow \boldsymbol{Z}+\boldsymbol{e}_{2}\right)=1$, we find $Z=4 / 3$, and finally

$$
\begin{aligned}
p\left(\boldsymbol{Z}-\boldsymbol{e}_{2} \mid \boldsymbol{Z}\right) & =\frac{1}{3}, \\
p\left(\boldsymbol{Z}+\boldsymbol{e}_{2} \mid \boldsymbol{Z}\right) & =\frac{2}{3} .
\end{aligned}
$$

- if the vacancy is on the backbone but not adjacent to the TP, we can adapt the previous arguments to write

$$
\begin{aligned}
& p\left(\boldsymbol{Z} \pm \boldsymbol{e}_{1} \mid \boldsymbol{Z}\right)=\frac{1}{6}, \\
& p\left(\boldsymbol{Z} \pm \boldsymbol{e}_{2} \mid \boldsymbol{Z}\right)=\frac{1}{3} .
\end{aligned}
$$

- if the vacancy is on the backbone and at the right of the TP (this is easily extended to the case where the vacancy is at the left of the TP), the same arguments lead to

$$
\begin{aligned}
& p\left(\boldsymbol{Z}-\boldsymbol{e}_{1} \mid \boldsymbol{Z}\right)=\frac{2}{7}, \\
& p\left(\boldsymbol{Z}+\boldsymbol{e}_{1} \mid \boldsymbol{Z}\right)=\frac{1}{7}, \\
& p\left(\boldsymbol{Z} \pm \boldsymbol{e}_{2} \mid \boldsymbol{Z}\right)=\frac{2}{7} .
\end{aligned}
$$

- if the vacancy is on a tooth adjacent to the TP, it steps away from the TP with probability 1 as the TP is constrained to remain on the backbone.

These evolution rules are summarized in Fig. 4. 


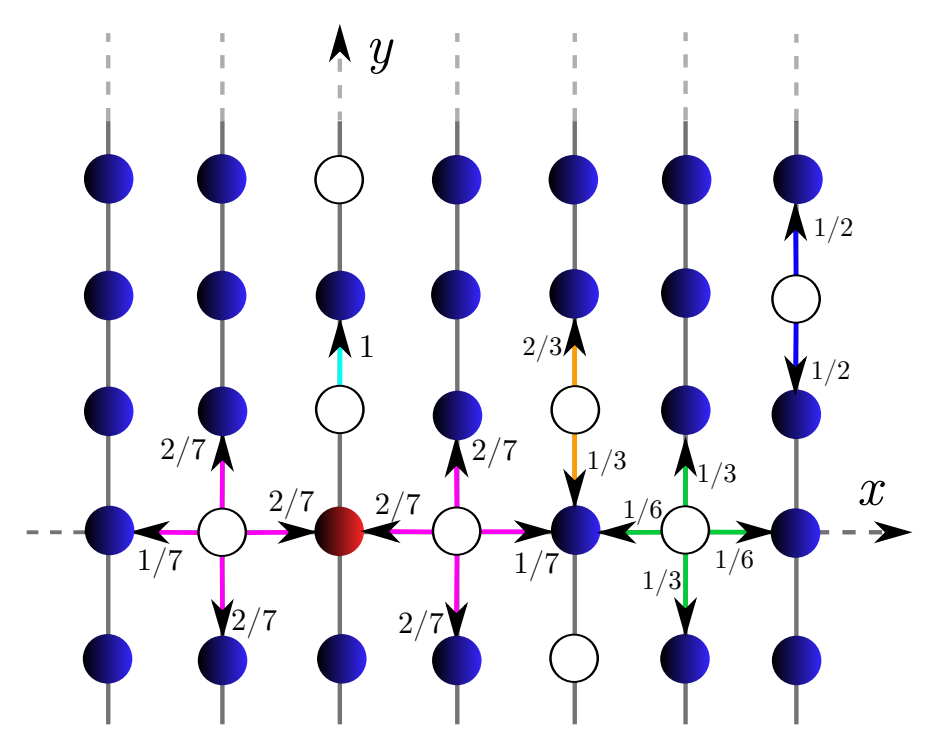

FIG. 4. Evolution rules of a vacancy on the two-dimensional comb.

\section{FIRST REGIME}

In the main text, we established the following expression for the generating function associated with the cumulant generating function of the position of the TP:

$$
\widehat{\Psi}(k ; \xi) \underset{\rho_{0} \rightarrow 0}{\sim}-2 \rho_{0} \frac{H(\xi)(1-\cos k)}{(1-\xi)\left(1+\widehat{F}_{1}\right)},
$$

where we use the simplified notation $\widehat{F}_{1} \equiv \widehat{F}\left(\mathbf{0} \mid e_{1} ; \xi\right)$, and where $\widehat{F}(\mathbf{0} \mid \boldsymbol{r} ; \xi)$ is the generating function associated with $F_{t}(\mathbf{0} \mid \boldsymbol{r})$, defined as the probability for a vacancy to reach the origin for the first time at time $t$ knowing that it started from site $\boldsymbol{r}$. We also introduced the quantity $H(\xi) \equiv \sum_{x=1}^{\infty} \sum_{y=-\infty}^{\infty} \widehat{F}(\mathbf{0} \mid x, y ; \xi)$. The determination of the cumulant generating function then relies on the determination of $H(\xi)$ and $\widehat{F}_{1}$. Detailed calculations of these quantities are given below.

\section{A. Calculation of $H(\xi)$}

The quantity $H(\xi)$ is defined as

$$
H(\xi)=\sum_{x=1}^{\infty} \sum_{y=-\infty}^{\infty} \widehat{F}(0,0 \mid x, y ; \xi)
$$

The first-passage densities $\widehat{F}(0,0 \mid x, y ; \xi)$ are related to the random walk of a vacancy on a lattice with specific and non-uniform transition rates, and represented in Fig. 4.

These first-passage densities can be calculated using the following fundamental relation, resulting from the tree-like structure of the two-dimensional comb, and valid for any site $\boldsymbol{r}^{\prime}$ belonging to the path from $\boldsymbol{r}$ to $\boldsymbol{r}_{0}$ [43]:

$$
\widehat{F}\left(\boldsymbol{r} \mid \boldsymbol{r}_{0} ; \xi\right)=\widehat{F}\left(\boldsymbol{r} \mid \boldsymbol{r}^{\prime} ; \xi\right) \widehat{F}\left(\boldsymbol{r}^{\prime} \mid \boldsymbol{r}_{0} ; \xi\right) .
$$

To compute $\widehat{F}(0,0 \mid x, y ; \xi)(x>0)$, we consider separately the situations where $y=0$ and $y \neq 0$, and obtain:

- if $y \neq 0$, the path from $(x, y)$ to $(0,0)$ is decomposed as follows:

$$
(x, y) \rightarrow(x, \operatorname{sgn}(y)) \rightarrow(x, 0) \rightarrow(1,0) \rightarrow(0,0)
$$


so that generating function of the first-passage density is

$$
\widehat{F}(0,0 \mid x, y ; \xi)=\widehat{F}(0,0 \mid 1,0 ; \xi) \widehat{F}(1,0 \mid x, 0 ; \xi) \widehat{F}(x, 0 \mid x, \operatorname{sgn}(y) ; \xi) \widehat{F}(x, \operatorname{sgn}(y) \mid x, y ; \xi)
$$

We introduce the quantities

$$
\begin{aligned}
f_{2,1}(\xi) & =\widehat{F}(1,0 \mid 2,0 ; \xi), \\
f_{2,2}(\xi) & =\widehat{F}(1,0 \mid 1,1 ; \xi) \\
f_{1,1}(\xi) & =\widehat{F}(1,1 \mid 1,2 ; \xi)
\end{aligned}
$$

The last quantity is relative to the random walk of a vacancy on a tooth far from the backbone, i.e. on a one-dimensional structure. It is then given by [34]:

$$
f_{1,1}(\xi)=\frac{1-\sqrt{1-\xi^{2}}}{\xi}
$$

Finally, using these relations and the fundamental equation (27) once again, we get

$$
\widehat{F}(0,0 \mid x, y ; \xi)=\widehat{F}_{1}\left[f_{2,1}(\xi)\right]^{|x|-1} f_{2,2}(\xi)\left[f_{1,1}(\xi)\right]^{|y|-1} .
$$

- if $y=0$, the path is simply decomposed as:

$$
(x, 0) \rightarrow(1,0) \rightarrow(0,0)
$$

and we find

$$
\begin{aligned}
\widehat{F}(0,0 \mid x, 0 ; \xi) & =\widehat{F}(0,0 \mid 1,0 ; \xi) \widehat{F}(1,0 \mid x, 0 ; \xi) \\
& =\widehat{F}_{1}\left[f_{2,1}(\xi)\right]^{|x|-1}
\end{aligned}
$$

We now go back to the calculation of $H(\xi)$,

$$
\begin{aligned}
H(\xi) & =\sum_{x=1}^{\infty} \sum_{y=-\infty}^{\infty} \widehat{F}(0,0 \mid x, y ; \xi) \\
& =\sum_{x=1}^{\infty}\left[\widehat{F}(0,0 \mid x, 0 ; \xi)+2 \sum_{y=1}^{\infty} \widehat{F}(0,0 \mid x, y ; \xi)\right] \\
& =\widehat{F}_{1} \sum_{x=1}^{\infty} f_{2,1}(\xi)^{x-1}\left[1+2 \sum_{y=1}^{\infty} f_{2,2}(\xi) f_{1,1}(\xi)^{y-1}\right] \\
& =\frac{\widehat{F}_{1}}{1-f_{2,1}(\xi)}\left(1+2 \frac{f_{2,2}(\xi)}{1-f_{1,1}(\xi)}\right)
\end{aligned}
$$

We then calculate separately $f_{2,2}(\xi)$ and $f_{2,1}(\xi)$, defined in Eqs. (30) and (31), which are relative to the random walk of a vacancy away from the tracer.

In what follows, we will use the general relation

$$
\widehat{F}\left(\boldsymbol{r} \mid \boldsymbol{r}_{0} ; \xi\right)=\xi \sum_{\boldsymbol{w}} \widehat{F}(\boldsymbol{r} \mid \boldsymbol{w} ; \xi) p\left(\boldsymbol{w} \mid \boldsymbol{r}_{0}\right)
$$

where $p\left(\boldsymbol{w} \mid \boldsymbol{r}_{0}\right)$ is the probability to jump from $\boldsymbol{r}_{0}$ to $\boldsymbol{w}$ in a single step, and where the sum runs over all lattice sites. This relation is obtained by partitioning over the first step of the walk. 
1. Calculation of $f_{2,2}(\xi)$

We use the general relation (42), and write

$$
\begin{aligned}
f_{2,2}(\xi) & =\xi \sum_{\boldsymbol{w}} \widehat{F}\left(\boldsymbol{e}_{1} \mid \boldsymbol{w} ; \xi\right) p\left(\boldsymbol{w} \mid \boldsymbol{e}_{1}+\boldsymbol{e}_{2}\right) \\
& =\xi\left[\widehat{F}\left(\boldsymbol{e}_{1} \mid \boldsymbol{e}_{1} ; \xi\right) p\left(\boldsymbol{e}_{1} \mid \boldsymbol{e}_{1}+\boldsymbol{e}_{2}\right)+\widehat{F}\left(\boldsymbol{e}_{1} \mid \boldsymbol{e}_{1}+2 \boldsymbol{e}_{2} ; \xi\right) p\left(\boldsymbol{e}_{1}+2 \boldsymbol{e}_{2} \mid \boldsymbol{e}_{1}+\boldsymbol{e}_{2}\right)\right]
\end{aligned}
$$

Because of the tree structure of the lattice, one writes

$$
\begin{aligned}
\widehat{F}\left(\boldsymbol{e}_{1} \mid \boldsymbol{e}_{1} ; \xi\right) & =1 \\
\widehat{F}\left(\boldsymbol{e}_{1} \mid \boldsymbol{e}_{1}+2 \boldsymbol{e}_{2} ; \xi\right) & =\widehat{F}\left(\boldsymbol{e}_{1} \mid \boldsymbol{e}_{1}+\boldsymbol{e}_{2} ; \xi\right) \widehat{F}\left(\boldsymbol{e}_{1}+\boldsymbol{e}_{2} \mid \boldsymbol{e}_{1}+2 \boldsymbol{e}_{2} ; \xi\right)=f_{2,2}(\xi) f_{1,1}(\xi) .
\end{aligned}
$$

Finally, we get the following equation for $f_{2,2}(\xi)$ :

$$
f_{2,2}(\xi)=\xi\left[\frac{1}{3}+\frac{2}{3} f_{1,1}(\xi) f_{2,2}(\xi)\right] .
$$

Solving it and recalling the expression of $f_{1,1}(\xi)$ (Eq. (33)), we obtain

$$
f_{2,2}(\xi)=\frac{\xi}{1+2 \sqrt{1-\xi^{2}}}
$$

\section{Calculation of $f_{2,1}(\xi)$}

Using again Eq. (42), we write

$$
\begin{aligned}
f_{2,1}(\xi) & =\xi \sum_{\boldsymbol{w}} \widehat{F}\left(\boldsymbol{e}_{1} \mid \boldsymbol{w} ; \xi\right) p\left(\boldsymbol{w} \mid 2 \boldsymbol{e}_{1}\right) \\
& =\xi\left[\widehat{F}\left(\boldsymbol{e}_{1} \mid \boldsymbol{e}_{1} ; \xi\right) p\left(\boldsymbol{e}_{1} \mid 2 \boldsymbol{e}_{1}\right)+\widehat{F}\left(\boldsymbol{e}_{1} \mid 3 \boldsymbol{e}_{1} ; \xi\right) p\left(3 \boldsymbol{e}_{1} \mid 2 \boldsymbol{e}_{1}\right)+2 \widehat{F}\left(\boldsymbol{e}_{1} \mid 2 \boldsymbol{e}_{1}+\boldsymbol{e}_{2} ; \xi\right) p\left(2 \boldsymbol{e}_{1}+\boldsymbol{e}_{2} \mid 2 \boldsymbol{e}_{1}\right)\right] \\
& =\xi\left[\frac{1}{6}+\frac{1}{6} f_{2,1}(\xi)^{2}+\frac{2}{3} f_{2,2}(\xi) f_{2,1}(\xi)\right] .
\end{aligned}
$$

$f_{2,1}(\xi)$ is then the solution of a second-order equation. Choosing the solution fulfilling the condition $f_{2,1}(0)=0$, we get

$$
f_{2,1}(\xi)=\frac{3}{\xi}-2 f_{2,2}(\xi)-\sqrt{\left(\frac{3}{\xi}-2 f_{2,2}(\xi)\right)^{2}-1}
$$

\section{B. Calculation of $\widehat{F}_{1}$}

In order to calculate the generating function $\widehat{F}_{1}=\widehat{F}\left(\mathbf{0} \mid e_{1} ; \xi\right)$, we consider the first passage density $F_{t}\left(\mathbf{0} \mid e_{1}\right)$ partitioned over the first time step

$$
F_{t}\left(\mathbf{0} \mid \boldsymbol{e}_{1}\right)=\frac{2}{7} \delta_{t, 1}+\frac{1}{7} \sum_{t^{\prime}=1}^{t} F_{t^{\prime}-1}\left(\boldsymbol{e}_{1} \mid 2 \boldsymbol{e}_{1}\right) F_{t-t^{\prime}}\left(\mathbf{0} \mid \boldsymbol{e}_{1}\right)+2 \times \frac{2}{7} \sum_{t^{\prime}=1}^{t} F_{t^{\prime}-1}\left(\boldsymbol{e}_{1} \mid \boldsymbol{e}_{1}+\boldsymbol{e}_{2}\right) F_{t-t^{\prime}}\left(\boldsymbol{e} \mid \boldsymbol{e}_{1}\right) .
$$

The first term of right-hand side corresponds to the situation where the vacancy jumps onto the origin of the lattice at the first step (i.e. in direction $-\boldsymbol{e}_{1}$ ). The second (resp. third) term corresponds to the situation where the first step of the vacancy is in direction $+\boldsymbol{e}_{1}\left(\right.$ resp. $\left.\pm \boldsymbol{e}_{2}\right)$. In terms of generating functions, we get

$$
\begin{aligned}
\widehat{F}_{1} & =\frac{\frac{2}{7} \xi}{1-\frac{1}{7} \xi \widehat{F}\left(\boldsymbol{e}_{1} \mid 2 \boldsymbol{e}_{1} ; \xi\right)-\frac{4}{7} \xi \widehat{F}\left(\boldsymbol{e}_{1} \mid \boldsymbol{e}_{1}+\boldsymbol{e}_{2} ; \xi\right)} \\
& =\frac{\frac{2}{7} \xi}{1-\frac{1}{7} \xi f_{2,1}(\xi)-\frac{4}{7} \xi f_{2,2}(\xi)} .
\end{aligned}
$$




\section{Long-time expansion of the cumulants}

Recalling the expression of $\widehat{\Psi}(k ; \xi)$ from Eq. (25):

$$
\widehat{\Psi}(k ; \xi) \underset{\rho_{0} \rightarrow 0}{\sim}-2 \rho_{0} \frac{H(\xi)(1-\cos k)}{(1-\xi)\left(1+\widehat{F}_{1}\right)},
$$

we write its expansion in powers of $k$ and obtain

$$
\widehat{\Psi}(k ; \xi) \underset{\rho_{0} \rightarrow 0}{\sim}-2 \rho_{0} \frac{H(\xi)}{(1-\xi)\left(1+\widehat{F}_{1}\right)} \sum_{n=1}^{\infty}(-1)^{n+1} \frac{k^{2 n}}{(2 n) !} .
$$

From the definition of the cumulant generating function $\widehat{\Psi}(k ; \xi)$, the coefficients of its expansion in powers of $k$ are related to the cumulants of $X_{t}$ as follows:

$$
\widehat{\Psi}(k ; \xi)=\sum_{n=0}^{\infty} \frac{(\mathrm{i} k)^{n}}{n !} \widehat{\kappa}^{(n)}(\xi) .
$$

Identifying the expansions (57) and (58), we obtain that all the even cumulants are equal and have the following expression

$$
\widehat{\kappa}^{(2 n)}(\xi) \underset{\rho_{0} \rightarrow 0}{\sim} \frac{2 \rho_{0} H(\xi)}{(1-\xi)\left(1+\widehat{F}_{1}\right)} .
$$

We also verify that, for symmetry reasons, the odd cumulants are null. Using the explicit expressions of $H(\xi)$, Eq. (41), and $\widehat{F}_{1}$, Eq. (55), we obtain the expansion of the even cumulants in the long-time limit $(\xi \rightarrow 1)$ :

$$
\lim _{\rho_{0} \rightarrow 0} \frac{\widehat{\kappa}^{(2 n)}(\xi)}{\rho_{0}} \underset{\xi \rightarrow 1}{\sim} \frac{1}{2^{5 / 4}(1-\xi)^{7 / 4}} .
$$

Using a Tauberian theorem, we retrieve the long-time limit of these cumulants, corresponding to Eq. (5) from the main text,

$$
\lim _{\rho_{0} \rightarrow 0} \frac{\kappa^{(2 n)}(t)}{\rho_{0}} \underset{t \rightarrow \infty}{\sim} \frac{1}{2^{5 / 4} \Gamma(7 / 4)} t^{3 / 4}
$$

\section{Influence of the initial conditions}

In the previous sections, we considered the situation where the vacancies are initially uniformly distributed on the lattice. The situation where the vacancies are initially located on the backbone of the structure can be studied with the formalism presented above. It is straightforward to show that, with this initial condition, the cumulant generating function becomes

$$
\widehat{\Psi}(k ; \xi) \underset{\rho_{0} \rightarrow 0}{\sim}-2 \rho_{0} \frac{H^{\prime}(\xi)(1-\cos k)}{(1-\xi)\left(1+\widehat{F}_{1}\right)},
$$

where

$$
H^{\prime}(\xi) \equiv \sum_{x=1}^{\infty} \widehat{F}(\mathbf{0} \mid x, y=0 ; \xi) .
$$

The expression of the even cumulants is deduced as in section II C, and we obtain

$$
\widehat{\kappa}^{(2 n)}(\xi) \underset{\rho_{0} \rightarrow 0}{\sim} \frac{2 \rho_{0} \widehat{F}_{1}}{(1-\xi)\left(1+\widehat{F}_{1}\right)\left[1-f_{2,1}(\xi)\right]} .
$$




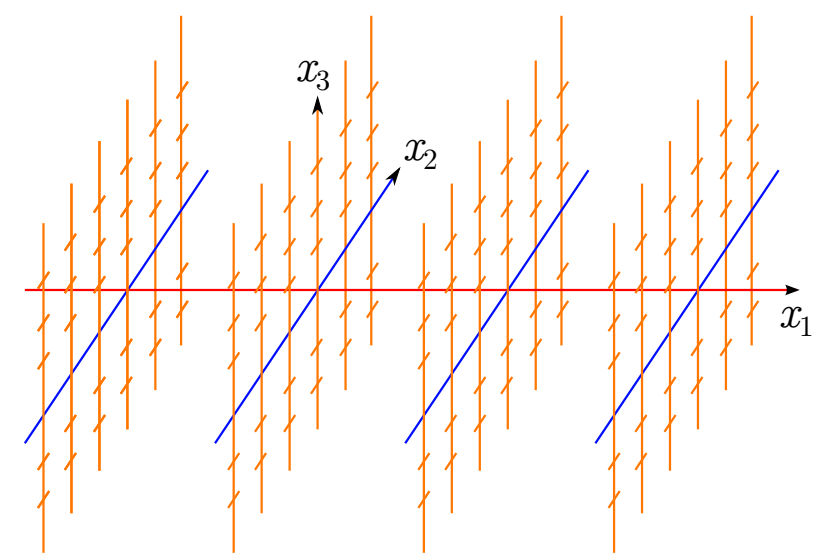

FIG. 5. Representation of the three-dimensional comb lattice.

Finally, recalling the expressions of $\widehat{F}_{1}$ (Eq. (55)) and $H^{\prime}(\xi)$ (Eq. (63)) in terms of the first-passage time densities computed in section II A, we obtain the long-time limit $(\xi \rightarrow 1)$ of the even cumulants:

$$
\lim _{\rho_{0} \rightarrow 0} \frac{\widehat{\kappa}^{(2 n)}(\xi)}{\rho_{0}} \underset{\xi \rightarrow 1}{\sim} \frac{1}{2^{7 / 4}(1-\xi)^{5 / 4}} .
$$

Using a Tauberian theorem, we retrieve the long-time limit of these cumulants:

$$
\lim _{\rho_{0} \rightarrow 0} \frac{\kappa^{(2 n)}(t)}{\rho_{0}} \underset{t \rightarrow \infty}{\sim} \frac{1}{2^{7 / 4} \Gamma(5 / 4)} t^{1 / 4}
$$

\section{E. Definition of a $d$-dimensional comb}

In the main text, we present the expression of the variance of the position of the TP on a generalized $d$-dimensional comb. This structure is defined recursively as follows: starting from $\mathbf{C}_{1}$ (a one-dimensional lattice), $\mathbf{C}_{d}$ is obtained from $\mathbf{C}_{d-1}$ by attaching at each point a two-way infinite path. We represent in Fig. 5 the three-dimensional comb.

\section{SECOND REGIME}

\section{A. Introduction}

The key point underlying the crossover to a second regime is that the previous analytical results are derived by taking the limit $\rho_{0} \rightarrow 0$ before the long time limit. In this case, to leading order, the TP does not move before being reached by a given vacancy. In fact, the TP actually diffuses due to its interactions with the other vacancies. Due to the effective diffusion of the TP, each vacancy is then assumed to have an additional jump probability in the $x$ direction, denoted by $D\left(\rho_{0}\right)$, and which vanishes when $\rho_{0} \rightarrow 0$. We will write for simplicity $\epsilon \equiv D\left(\rho_{0}\right)$. The vacancies then perform two-dimensional random walks. We consider simplified evolution rules of the vacancies, which are expected to give a correct qualitative description of the system. The evolution rules for the vacancies are represented in Fig. 6.

\section{B. Single-vacancy problem}

As in the previous calculation, we first consider the situation where there is only one vacancy on the lattice. We denote by $P_{t}^{(1)}\left(\boldsymbol{X} \mid \boldsymbol{Y}_{0} ; \epsilon\right)$ the probability to find the TP at position $\boldsymbol{X}$ at time $t$ knowing that the vacancy started from site $\boldsymbol{Y}_{0}$. This probability can be written in terms of the first-passage time densities $F_{t}(\mathbf{0} \mid \boldsymbol{r} ; \epsilon)$ (probability for a vacancy to reach the origin for the first time at time $t$ starting from $\boldsymbol{r}$ ) and the conditional first-passage densities $F_{t}^{*}\left(\mathbf{0}\left|\boldsymbol{e}_{\mu}\right| \boldsymbol{r} ; \epsilon\right)$ (probability for a vacancy to reach the origin for the first time at time $t$ starting from $\boldsymbol{r}$ and being at 


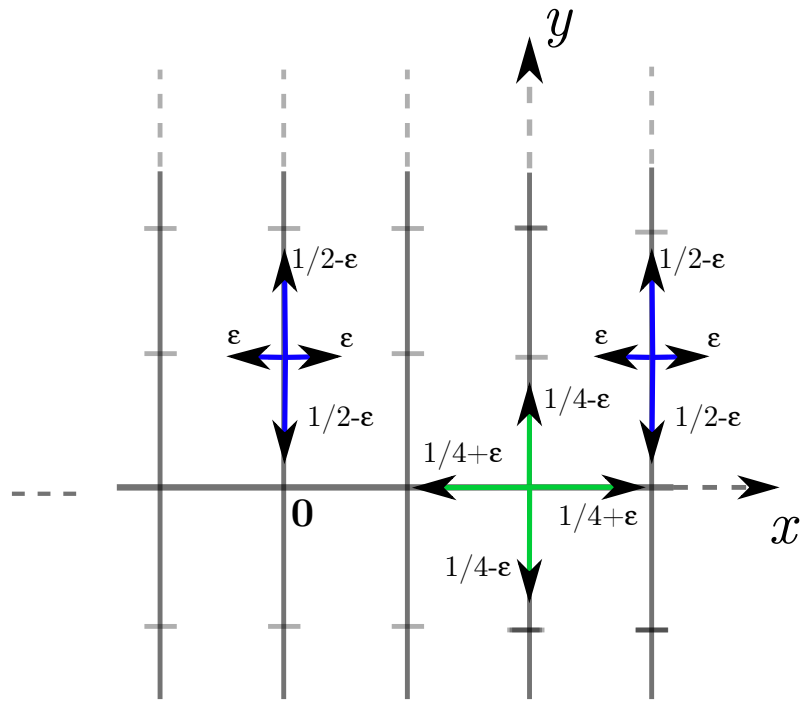

FIG. 6. Simplified evolution rules of the vacancies, when they experience an additional jump probability $\epsilon \equiv D\left(\rho_{0}\right)$.

site $\boldsymbol{e}_{\mu}$ at time $\left.t-1\right)$. Summing over the number of steps $p$ taken by the TP, over the directions $\nu_{1}, \ldots, \nu_{p}$ of these $p$ steps and over the length of the time intervals $m_{j}$ elapsed between the $(j-1)$-th and $j$-th step, we get

$$
\begin{aligned}
P_{t}^{(1)}\left(\boldsymbol{X} \mid \boldsymbol{Y}_{0} ; \epsilon\right) & =\delta_{\boldsymbol{X}, \mathbf{0}}\left(1-\sum_{j=0}^{t} F_{j}\left(\mathbf{0} \mid \boldsymbol{Y}_{0} ; \epsilon\right)\right) \\
& +\sum_{p=1}^{\infty} \sum_{m_{1}=1}^{\infty} \ldots \sum_{m_{p}=1}^{\infty} \sum_{m_{p+1}=0}^{\infty} \delta_{m_{1}+\ldots+m_{p+1}, t} \sum_{\nu_{1}} \ldots \sum_{\nu_{p}} \delta_{\mathbf{e}_{\nu_{1}}+\ldots+\mathbf{e}_{\nu_{p}}, \boldsymbol{X}} \\
& \times\left(1-\sum_{j=0}^{m_{p+1}} F_{j}\left(\mathbf{0} \mid-\boldsymbol{e}_{\nu_{p}} ; \epsilon\right)\right) F_{m_{p}}^{*}\left(\mathbf{0}\left|\boldsymbol{e}_{\nu_{p}}\right|-\boldsymbol{e}_{\nu_{p-1}} ; \epsilon\right) \ldots F_{m_{2}}^{*}\left(\mathbf{0}\left|\boldsymbol{e}_{\nu_{2}}\right|-\boldsymbol{e}_{\nu_{1}} ; \epsilon\right) F_{m_{1}}^{*}\left(\mathbf{0}\left|\boldsymbol{e}_{\nu_{1}}\right| \boldsymbol{Y}_{0} ; \epsilon\right) .
\end{aligned}
$$

The Fourier-Laplace transform of Eq. (67) writes

$$
\widetilde{\widehat{P}}^{(1)}(k \mid \boldsymbol{Z} ; \xi, \epsilon)=\frac{1}{1-\xi}\left[1+\frac{1}{\mathcal{D}(k ; \xi, \epsilon)} \sum_{b= \pm 1} U_{b}(k ; \xi) F^{*}\left(\mathbf{0}\left|\boldsymbol{e}_{b}\right| \boldsymbol{Z} ; \xi, \epsilon\right)\right],
$$

where we defined:

$$
\begin{aligned}
\mathcal{D}(k ; \xi, \epsilon) & =\operatorname{det}[\mathbf{1}-T(k ; \xi, \epsilon)] \\
T(k ; \xi, \epsilon) & =\left(\begin{array}{cc}
\mathrm{e}^{\mathrm{i} k} \widehat{F}^{*}\left(\mathbf{0}\left|\boldsymbol{e}_{1}\right| \boldsymbol{e}_{-1} ; \xi, \epsilon\right) & \mathrm{e}^{\mathrm{i} k} \widehat{F}^{*}\left(\mathbf{0}\left|\boldsymbol{e}_{1}\right| \boldsymbol{e}_{1} ; \xi, \epsilon\right) \\
\mathrm{e}^{-\mathrm{i} k} \widehat{F}^{*}\left(\mathbf{0}\left|\boldsymbol{e}_{-1}\right| \boldsymbol{e}_{-1} ; \xi, \epsilon\right) & \mathrm{e}^{-\mathrm{i} k} \widehat{F}^{*}\left(\mathbf{0}\left|\boldsymbol{e}_{-1}\right| \boldsymbol{e}_{1} ; \xi, \epsilon\right)
\end{array}\right) \\
U_{b}(k ; \xi, \epsilon) & =\mathcal{D}(k ; \xi, \epsilon) \sum_{a= \pm 1}\left(1-\mathrm{e}^{-\mathrm{i} a k}\right)\left[(\mathbf{1}-T(k ; \xi, \epsilon))^{-1}\right]_{a, b} \mathrm{e}^{\mathrm{i} b k} .
\end{aligned}
$$

The TP being symmetric, we have the following relations

$$
\begin{aligned}
\widehat{F}^{*}\left(\mathbf{0}\left|\boldsymbol{e}_{1}\right| \boldsymbol{e}_{1} ; \xi, \epsilon\right) & =\widehat{F}^{*}\left(\mathbf{0}\left|\boldsymbol{e}_{-1}\right| \boldsymbol{e}_{-1} ; \xi, \epsilon\right) \\
\widehat{F}^{*}\left(\mathbf{0}\left|\boldsymbol{e}_{1}\right| \boldsymbol{e}_{-1} ; \xi, \epsilon\right) & =\widehat{F}^{*}\left(\mathbf{0}\left|\boldsymbol{e}_{-1}\right| \boldsymbol{e}_{1} ; \xi, \epsilon\right),
\end{aligned}
$$

so that $\mathcal{D}(k ; \xi, \epsilon)$ and $U_{ \pm 1}(k ; \xi, \epsilon)$ reduce to:

$$
\begin{aligned}
\mathcal{D}(k ; \xi, \epsilon) & =1+\widehat{F}^{*}\left(\mathbf{0}\left|\boldsymbol{e}_{1}\right| \boldsymbol{e}_{-1} ; \xi, \epsilon\right)^{2}-\widehat{F}^{*}\left(\mathbf{0}\left|\boldsymbol{e}_{1}\right| \boldsymbol{e}_{1} ; \xi, \epsilon\right)^{2}-2 \widehat{F}^{*}\left(\mathbf{0}\left|\boldsymbol{e}_{1}\right| \boldsymbol{e}_{-1} ; \xi, \epsilon\right) \cos k, \\
U_{ \pm 1}(k ; \xi, \epsilon) & =\left(\mathrm{e}^{ \pm \mathrm{i} k}-1\right)\left[1-\mathrm{e}^{\mp \mathrm{i} k} \widehat{F}^{*}\left(\mathbf{0}\left|\boldsymbol{e}_{1}\right| \boldsymbol{e}_{-1} ; \xi, \epsilon\right)-\widehat{F}^{*}\left(\mathbf{0}\left|\boldsymbol{e}_{1}\right| \boldsymbol{e}_{1} ; \xi, \epsilon\right)\right] .
\end{aligned}
$$


We finally obtain the following expression of the single vacancy propagator in terms of the first-passage time densities $\widehat{F}^{*}$ :

$$
\widetilde{\widehat{P}}^{(1)}(k \mid \boldsymbol{Z} ; \xi, \epsilon)=\frac{1}{1-\xi}\left[1+\frac{\sum_{\nu= \pm 1} F^{*}\left(\mathbf{0}\left|\boldsymbol{e}_{\nu}\right| \boldsymbol{Z} ; \xi, \epsilon\right)\left(\mathrm{e}^{\nu \mathrm{i} k}-1\right)\left(1-\mathrm{e}^{-\nu \mathrm{i} k} \widehat{F}_{-1}^{*}-\widehat{F}_{1}^{*}\right)}{1-\left(\widehat{F}_{1}^{*}\right)^{2}+\left(\widehat{F}_{-1}^{*}\right)^{2}-2 \widehat{F}_{-1}^{*} \cos k}\right]
$$

where we introduced the simplified notation

$$
\widehat{F}_{\nu}^{*} \equiv F^{*}\left(\mathbf{0}\left|e_{1}\right| e_{\nu} ; \xi, \epsilon\right)
$$

\section{Finite density of vacancies}

As for the study of the first regime, we now assume that the density of vacancies is equal to $\rho_{0}$, and we show that the Fourier-Laplace transform of the cumulant generating function in the limit $\rho_{0} \rightarrow 0$ is given by

$$
\widehat{\Psi}(k ; \xi, \epsilon) \underset{\rho_{0} \rightarrow 0}{\sim}-\rho_{0} \sum_{a= \pm 1}\left[\frac{1}{1-\xi}-\widehat{\widetilde{P}}^{(1)}\left(k \mid \boldsymbol{e}_{-a} ; \xi, \epsilon\right) \mathrm{e}^{\mathrm{i} a k}\right] \sum_{\boldsymbol{Z} \neq \mathbf{0}} F^{*}\left(\mathbf{0}\left|\boldsymbol{e}_{a}\right| \boldsymbol{Z} ; \xi, \epsilon\right) .
$$

The TP being symmetric, the quantity $\sum_{\boldsymbol{Z} \neq \mathbf{0}} F^{*}\left(\mathbf{0}\left|\boldsymbol{e}_{a}\right| \boldsymbol{Z} ; \xi, \epsilon\right)$ is independent of $a$,

$$
\sum_{Z \neq \mathbf{0}} F^{*}\left(\mathbf{0}\left|e_{-1}\right| Z ; \xi, \epsilon\right)=\sum_{Z \neq 0} F^{*}\left(\mathbf{0}\left|e_{1}\right| Z ; \xi, \epsilon\right)
$$

and we obtain

$$
\widehat{\Psi}(k ; \xi) \underset{\rho_{0} \rightarrow 0}{\sim}-\rho_{0}\left(\sum_{\boldsymbol{Z} \neq \mathbf{0}} F^{*}\left(\mathbf{0}\left|\boldsymbol{e}_{1}\right| \boldsymbol{Z} ; \xi, \epsilon\right)\right) \sum_{a= \pm 1}\left[\frac{1}{1-\xi}-\widehat{\widetilde{P}}^{(1)}\left(k \mid \boldsymbol{e}_{-a} ; \xi, \epsilon\right) \mathrm{e}^{\mathrm{i} a k}\right] .
$$

Defining $\Sigma(\xi, \epsilon)=\sum_{\boldsymbol{Z} \neq \mathbf{0}} F^{*}\left(\mathbf{0}\left|\boldsymbol{e}_{1}\right| \boldsymbol{Z} ; \xi, \epsilon\right)$, we finally have

$$
\widehat{\Psi}(k ; \xi, \epsilon) \underset{\rho_{0} \rightarrow 0}{\sim}-\rho_{0} \Sigma(\xi, \epsilon) \sum_{a= \pm 1}\left[\frac{1}{1-\xi}-\widehat{\widetilde{P}}^{(1)}\left(k \mid \boldsymbol{e}_{-a} ; \xi, \epsilon\right) \mathrm{e}^{\mathrm{i} a k}\right]
$$

The expression of the variance of the position of the TP is easily deduced from

$$
\widehat{\kappa}^{(2)}(\xi, \epsilon)=-\frac{\partial^{2} \widehat{\Psi}(k ; \xi, \epsilon)}{\partial k^{2}}
$$

Using Eq. (76), we get

$$
\widehat{\kappa}^{(2)}(\xi, \epsilon)=-2 \rho_{0} \Sigma(\xi, \epsilon) \frac{\widehat{F}_{1}^{*}-\widehat{F}_{-1}^{*}-1}{\left(\widehat{F}_{1}^{*}-1+\widehat{F}_{-1}^{*}\right)\left(\widehat{F}_{1}^{*}+1-\widehat{F}_{-1}^{*}\right)}
$$

Consequently, the determination of the variance of the $\mathrm{TP}$ position only relies on the estimation on the following quantities:

- the $\operatorname{sum} \Sigma(\xi, \epsilon)=\sum_{\boldsymbol{Z} \neq \mathbf{0}} F^{*}\left(\mathbf{0}\left|\boldsymbol{e}_{1}\right| \boldsymbol{Z} ; \xi, \epsilon\right)$,

- the first-passage time densities $\widehat{F}_{ \pm 1}^{*}=\widehat{F}^{*}\left(\mathbf{0}\left|\boldsymbol{e}_{1}\right| \boldsymbol{e}_{ \pm 1} ; \xi, \epsilon\right)$.

In what follows, we evaluate the first-passage time densities $\widehat{F}_{ \pm 1}^{*}$ as well as the sum $\Sigma(\xi, \epsilon)$. 


\section{Evaluation of $\widehat{F}^{*}\left(\mathbf{0}\left|\boldsymbol{e}_{1}\right| \boldsymbol{e}_{ \pm 1} ; \xi, \epsilon\right)$}

In what follows, we write the conditional first-passage densities $\widehat{F}^{*}\left(\mathbf{0}\left|\boldsymbol{e}_{1}\right| \boldsymbol{e}_{ \pm 1} ; \xi, \epsilon\right)$ in terms of the propagators of the random walk of a vacancy with the rules specified in Fig. 6 . We first aim at computing $\widehat{F}^{*}\left(\mathbf{0}\left|\boldsymbol{e}_{1}\right| \boldsymbol{e}_{-1} ; \xi, \epsilon\right)$. We then assume that the vacancy starts at site $\boldsymbol{e}_{-1}$, and we write the following partition over the visits of the vacancy to the origin of the lattice:

$$
p\left(\boldsymbol{e}_{1} \rightarrow \mathbf{0}\right) P_{t-1}\left(\boldsymbol{e}_{1} \mid \boldsymbol{e}_{-1} ; \epsilon\right)=F_{t}^{*}\left(\mathbf{0}\left|\boldsymbol{e}_{1}\right| \boldsymbol{e}_{-1} ; \epsilon\right)+\sum_{t^{\prime}=0}^{t-1} p\left(\boldsymbol{e}_{1} \rightarrow \mathbf{0}\right) F_{t^{\prime}}\left(\mathbf{0} \mid \boldsymbol{e}_{-1} ; \epsilon\right) P_{t-1-t^{\prime}}\left(\boldsymbol{e}_{-1} \mid \mathbf{0} ; \epsilon\right)
$$

where $p\left(\boldsymbol{r} \rightarrow \boldsymbol{r}^{\prime}\right)$ is the probability for a vacancy to jump from site $\boldsymbol{r}$ to site $\boldsymbol{r}^{\prime}$ in a single step $\left(\right.$ here $\left.p\left(\boldsymbol{e}_{1} \rightarrow \mathbf{0}\right)=1 / 4+\epsilon\right)$. Multiplying the previous equation by $\xi^{t}$ and summing for $t$ going from 0 to $\infty$, we obtain the following relation between the associated generating functions:

$$
\xi\left(\frac{1}{4}+\epsilon\right) \widehat{P}\left(\boldsymbol{e}_{1} \mid \boldsymbol{e}_{-1} ; \xi, \epsilon\right)=\widehat{F}^{*}\left(\mathbf{0}\left|\boldsymbol{e}_{1}\right| \boldsymbol{e}_{-1} ; \xi, \epsilon\right)+\xi\left(\frac{1}{4}+\epsilon\right) \widehat{F}\left(\mathbf{0} \mid \boldsymbol{e}_{-1} ; \xi, \epsilon\right) \widehat{P}\left(\boldsymbol{e}_{1} \mid \mathbf{0} ; \xi, \epsilon\right)
$$

The first-passage density $\widehat{F}\left(\mathbf{0} \mid \boldsymbol{e}_{-1} ; \xi, \epsilon\right)$ is evaluated with the following renewal equation [34]:

$$
\widehat{F}\left(\mathbf{0} \mid e_{-1} ; \xi, \epsilon\right)=\frac{\widehat{P}\left(\mathbf{0} \mid e_{-1} ; \xi, \epsilon\right)}{\widehat{P}(\mathbf{0} \mid \mathbf{0} ; \xi, \epsilon)} .
$$

We obtain the following expression of $\widehat{F}^{*}\left(\mathbf{0}\left|\boldsymbol{e}_{1}\right| \boldsymbol{e}_{-1} ; \xi, \epsilon\right)$ in terms of the propagators $\widehat{P}$ :

$$
\widehat{F}^{*}\left(\mathbf{0}\left|\boldsymbol{e}_{1}\right| \boldsymbol{e}_{-1} ; \xi, \epsilon\right)=\xi\left(\frac{1}{4}+\epsilon\right)\left[\widehat{P}\left(\boldsymbol{e}_{1} \mid \boldsymbol{e}_{-1} ; \xi, \epsilon\right)-\frac{\widehat{P}\left(\mathbf{0} \mid \boldsymbol{e}_{-1} ; \xi, \epsilon\right) \widehat{P}\left(\boldsymbol{e}_{1} \mid \mathbf{0} ; \xi, \epsilon\right)}{\widehat{P}(\mathbf{0} \mid \mathbf{0} ; \xi, \epsilon)}\right]
$$

The random walk performed by the vacancy being translationally invariant in the direction of the backbone, we will use the following relations:

$$
\begin{aligned}
\widehat{P}\left(\boldsymbol{e}_{1} \mid \boldsymbol{e}_{-1} ; \xi, \epsilon\right) & =\widehat{P}\left(2 \boldsymbol{e}_{1} \mid \mathbf{0} ; \xi, \epsilon\right) \\
\widehat{P}\left(\mathbf{0} \mid \boldsymbol{e}_{-1} ; \xi, \epsilon\right) & =\widehat{P}\left(\boldsymbol{e}_{1} \mid \mathbf{0} ; \xi, \epsilon\right)
\end{aligned}
$$

We define $\alpha(\boldsymbol{r} ; \xi, \epsilon)$ as

$$
\alpha(\boldsymbol{r} ; \xi, \epsilon) \equiv \widehat{P}(\mathbf{0} \mid \mathbf{0} ; \xi, \epsilon)-\widehat{P}(\boldsymbol{r} \mid \mathbf{0} ; \xi, \epsilon)
$$

Then, the conditional first-passage densities are simply expressed in terms of $\alpha\left(\boldsymbol{e}_{1} ; \xi, \epsilon\right), \alpha\left(2 \boldsymbol{e}_{1} ; \xi, \epsilon\right)$ and $\widehat{P}(\mathbf{0} \mid \mathbf{0} ; \xi, \epsilon)$ :

$$
\widehat{F}^{*}\left(\mathbf{0}\left|\boldsymbol{e}_{1}\right| \boldsymbol{e}_{-1} ; \xi, \epsilon\right)=\xi\left(\frac{1}{4}+\epsilon\right)\left[2 \alpha\left(\boldsymbol{e}_{1} ; \xi, \epsilon\right)-\alpha\left(\boldsymbol{e}_{1} ; \xi, \epsilon\right)-\frac{\alpha\left(\boldsymbol{e}_{1} ; \xi, \epsilon\right)^{2}}{\widehat{P}(\mathbf{0} \mid \mathbf{0} ; \xi, \epsilon)}\right]
$$

A similar calculation leads to the following expression of $\widehat{F}^{*}\left(\mathbf{0}\left|\boldsymbol{e}_{1}\right| \boldsymbol{e}_{1} ; \xi, \epsilon\right)$ in terms of $\alpha\left(\boldsymbol{e}_{1} ; \xi, \epsilon\right)$ and $\widehat{P}(\mathbf{0} \mid \mathbf{0} ; \xi, \epsilon)$ :

$$
\widehat{F}^{*}\left(\mathbf{0}\left|\boldsymbol{e}_{1}\right| \boldsymbol{e}_{1} ; \xi, \epsilon\right)=\xi\left(\frac{1}{4}+\epsilon\right)\left[2 \alpha\left(\boldsymbol{e}_{1} ; \xi, \epsilon\right)-\frac{\alpha\left(\boldsymbol{e}_{1} ; \xi, \epsilon\right)^{2}}{\widehat{P}(\mathbf{0} \mid \mathbf{0} ; \xi, \epsilon)}\right]
$$

In what follows, we compute the quantities $\alpha\left(\boldsymbol{e}_{1} ; \xi, \epsilon\right), \alpha\left(2 \boldsymbol{e}_{1} ; \xi, \epsilon\right)$ and $\widehat{P}(\mathbf{0} \mid \mathbf{0} ; \xi, \epsilon)$ associated with the random walk of a vacancy with the evolution rules presented in Fig. 6. We follow the method first introduced by Nieuwenhuizen et al. [36].

For generality, we assume that if the vacancy is on the backbone (respectively not on the backbone), it has a probability $p_{b}^{\|}$(resp. $\left.p^{\|}\right)$to go left or right, and $p_{b}^{\perp}\left(\right.$ resp. $\left.p^{\perp}\right)$ to go up or down. Let $P_{x, y}(t)$ be the probability to find the vacancy at site $(x, y)$ at time $t$, knowing that it started from the origin. The master equations of the problem are the following:

- if $y \neq 0, \pm 1$ :

$$
P_{x, y}(t+1)=p^{\|}\left[P_{x+1, y}(t)+P_{x-1, y}(t)\right]+p^{\perp}\left[P_{x, y+1}(t)+P_{x, y-1}(t)\right]
$$


- if $y=0$ :

$$
P_{x, 0}(t+1)=p^{\perp}\left[P_{x, 1}(t)+P_{x,-1}(t)\right]+p_{b}^{\|}\left[P_{x+1,0}(t)+P_{x-1,0}(t)\right]
$$

- if $y= \pm 1$ :

$$
P_{x, \pm 1}(t+1)=p^{\perp} P_{x, \pm 2}(t)+p_{b}^{\perp} P_{x, 0}(t)+p^{\|}\left[P_{x+1, \pm 1}(t)+P_{x-1, \pm 1}(t)\right] .
$$

We introduce the following generating functions and Laplace transforms:

$$
\begin{aligned}
P_{b}\left(k_{x} ; \xi\right) & \equiv \sum_{t=0}^{\infty} \sum_{x=-\infty}^{\infty} \mathrm{e}^{\mathrm{i} k_{x} x} P_{x, 0}(t) \xi^{t}, \\
P\left(k_{x}, k_{y} ; \xi\right) & \equiv \sum_{t=0}^{\infty} \sum_{x, y=-\infty}^{\infty} \mathrm{e}^{\mathrm{i} k_{x} x} \mathrm{e}^{\mathrm{i} k_{y} y} P_{x, y}(t) \xi^{t} .
\end{aligned}
$$

Using the master equations, it is straightforward to show that $P$ and $P_{b}$ are related by

$$
P\left(k_{x}, k_{y} ; \xi\right)=\frac{1+2 \xi\left[\left(p_{b}^{\perp}-p^{\perp}\right) \cos k_{y}+\left(p_{b}^{\|}-p^{\|}\right) \cos k_{x}\right] P_{b}\left(k_{x} ; \xi\right)}{1-2 \xi\left[p^{\perp} \cos k_{y}+p^{\|} \cos k_{x}\right]} .
$$

In order to get an equation for $P_{b}\left(k_{x} ; \xi\right)$, we integrate each side of this equation over $k_{y}$ and use the simple relation between $P$ and $P_{b}$ :

$$
P_{b}\left(k_{x} ; \xi\right)=\int_{0}^{2 \pi} \frac{\mathrm{d} k_{y}}{2 \pi} P\left(k_{x}, k_{y} ; \xi\right) .
$$

We also use the following useful integrals:

$$
\begin{aligned}
& \int_{0}^{2 \pi} \frac{\mathrm{d} k}{2 \pi} \frac{1}{\cosh \mu-\cos k}=\frac{1}{\sinh \mu} \\
& \int_{0}^{2 \pi} \frac{\mathrm{d} k}{2 \pi} \frac{\cos k}{\cosh \mu-\cos k}=\frac{\mathrm{e}^{-\mu}}{\sinh \mu}
\end{aligned}
$$

to finally obtain

$$
P_{b}\left(k_{x} ; \xi\right)=\frac{1}{2 \xi p^{\perp} \sinh \mu-2 \xi\left[\left(p_{b}^{\perp}-p^{\perp}\right) \mathrm{e}^{-\mu}+\left(p_{b}^{\|}-p^{\|}\right) \cos k_{x}\right]},
$$

where $\mu$ is such that

$$
\cosh \mu=\frac{1-2 \xi p^{\|} \cos k_{x}}{2 \xi p^{\perp}}
$$

In what follows, we will use the explicit expressions of the transition probabilities:

$$
\begin{aligned}
p^{\|} & =\epsilon, \\
p^{\perp} & =\frac{1}{2}-\epsilon, \\
p_{b}^{\|} & =\frac{1}{4}+\epsilon, \\
p_{b}^{\perp} & =\frac{1}{4}-\epsilon .
\end{aligned}
$$

a. Computation of $\alpha\left(2 e_{1} ; \xi, \epsilon\right)$ We focus on the computation of $\alpha\left(2 e_{1} ; \xi, \epsilon\right)$, defined by:

$$
\begin{aligned}
\alpha\left(2 \boldsymbol{e}_{1} ; \xi, \epsilon\right) & =\widehat{P}(\mathbf{0} \mid \mathbf{0} ; \xi, \epsilon)-\widehat{P}\left(2 \boldsymbol{e}_{1} \mid \mathbf{0} ; \xi, \epsilon\right) \\
& =\int_{0}^{2 \pi} \frac{\mathrm{d} k_{x}}{2 \pi}\left(1-\mathrm{e}^{-2 \mathrm{i} k_{x}}\right) P_{b}\left(k_{x} ; \xi\right) .
\end{aligned}
$$


In the limit where we first take $\xi=1$, one has:

$$
P_{b}\left(k_{x} ; \xi=1\right)=\frac{2(1-2 \epsilon)}{1-\cos k_{x}+2 \sqrt{\epsilon}(1-4 \epsilon) \sqrt{\left(1-\cos k_{x}\right)\left(1-\epsilon-\epsilon \cos k_{x}\right)}}
$$

and consequently

$$
\alpha\left(2 e_{1} ; \xi=1, \epsilon\right)=\int_{0}^{2 \pi} \frac{\mathrm{d} k_{x}}{2 \pi} \frac{2(1-2 \epsilon)\left(1-\mathrm{e}^{-2 \mathrm{i} k_{x}}\right)}{1-\cos k_{x}+2 \sqrt{\epsilon}(1-4 \epsilon) \sqrt{\left(1-\cos k_{x}\right)\left(1-\epsilon-\epsilon \cos k_{x}\right)}} .
$$

For symmetry reasons, the imaginary part of the integral is zero, and

$$
\alpha\left(2 e_{1} ; \xi=1, \epsilon\right)=\frac{4}{\pi} \int_{0}^{\pi} \mathrm{d} k_{x} \frac{(1-2 \epsilon) \sin ^{2} k_{x}}{1-\cos k_{x}+2 \sqrt{\epsilon}(1-4 \epsilon) \sqrt{\left(1-\cos k_{x}\right)\left(1-\epsilon-\epsilon \cos k_{x}\right)}} .
$$

With the change of variable $u=\cos k_{x}$, one gets

$$
\begin{aligned}
\alpha\left(2 e_{1} ; \xi=1, \epsilon\right) & =\frac{4}{\pi}(1-2 \epsilon) \int_{-1}^{1} \mathrm{~d} u \frac{\sqrt{1-u^{2}}}{1-u+2 \sqrt{\epsilon}(1-4 \epsilon) \sqrt{(1-u)(1-\epsilon-\epsilon u)}} \\
& =\frac{4}{\pi}(1-2 \epsilon) \int_{-1}^{1} \mathrm{~d} u \frac{\sqrt{1+u}}{\sqrt{1-u}+2 \sqrt{\epsilon}(1-4 \epsilon) \sqrt{1-\epsilon-\epsilon u}} .
\end{aligned}
$$

We aim to compute the expansion of $\alpha\left(2 \boldsymbol{e}_{1} \mid \mathbf{0} ; \xi=1, \epsilon\right)$ in powers of $\epsilon$. The integral in the previous equation have an explicit expression:

$$
\begin{aligned}
\alpha\left(2 \boldsymbol{e}_{1} ; \xi=1, \epsilon\right)= & \frac{4(1-2 \epsilon)}{\pi(2 \epsilon-1)\left(32 \epsilon^{3}+2 \epsilon+1\right)^{2}}\left[256 \sqrt{2} \sqrt{(1-2 \epsilon) \epsilon^{9}}-64 \sqrt{2} \sqrt{(1-2 \epsilon) \epsilon^{7}} 16 \sqrt{2} \sqrt{(1-2 \epsilon) \epsilon^{5}}+32 \pi \epsilon^{3}\right. \\
& +4 \sqrt{2} \sqrt{(1-2 \epsilon) \epsilon^{3}}+2 \mathrm{i} \sqrt{2} \pi \sqrt{16 \epsilon^{3}+\epsilon}-32 \pi \epsilon^{2}-2(1-4 \epsilon)^{2}\left(8 \epsilon^{2}+2 \epsilon+1\right) \arctan \left(\sqrt{2} \sqrt{\frac{\epsilon}{1-2 \epsilon}}\right) \\
& -8 \mathrm{i} \sqrt{2} \pi \sqrt{16 \epsilon^{5}+\epsilon^{3}}+4 \sqrt{2}\left(\sqrt{16 \epsilon^{3}+\epsilon}-4 \sqrt{16 \epsilon^{5}+\epsilon^{3}}\right) \operatorname{arctanh}\left(\frac{1}{\sqrt{-32 \epsilon^{3}+16 \epsilon^{2}-2 \epsilon+1}}\right) \\
& +2 \pi \epsilon-2 \sqrt{2} \sqrt{(1-2 \epsilon) \epsilon}-\pi] .
\end{aligned}
$$

Its expansion for $\epsilon \rightarrow 0$ leads to:

$$
\alpha\left(2 e_{1} ; \xi=1, \epsilon\right) \underset{\epsilon \rightarrow 0}{=} 4+\frac{8 \sqrt{2}}{\pi} \sqrt{\epsilon} \ln \epsilon+\cdots
$$

b. Computation of $\alpha\left(\boldsymbol{e}_{1} \mid \mathbf{0} ; \xi=1, \epsilon\right)$ Similarly, we study $\alpha\left(\boldsymbol{e}_{1} \mid \mathbf{0} ; \xi=1, \epsilon\right)$, defined by

$$
\begin{aligned}
\alpha\left(\boldsymbol{e}_{1} ; \xi, \epsilon\right) & =\widehat{P}(\mathbf{0} \mid \mathbf{0} ; \xi, \epsilon)-\widehat{P}\left(\boldsymbol{e}_{1} \mid \mathbf{0} ; \xi, \epsilon\right) \\
& =\int_{0}^{2 \pi} \frac{\mathrm{d} k_{x}}{2 \pi}\left(1-\mathrm{e}^{-\mathrm{i} k_{x}}\right) P_{b}\left(k_{x} ; \xi\right) .
\end{aligned}
$$

Using again Eq. (110), and with the change of variable $u=\cos k_{x}$, we obtain

$$
\alpha\left(\boldsymbol{e}_{1} ; \xi, \epsilon\right)=\frac{2}{\pi}(1-2 \epsilon) \int_{-1}^{1} \mathrm{~d} u \frac{1}{\sqrt{1-u^{2}}+2 \sqrt{\epsilon}(1-4 \epsilon) \sqrt{(1+u)(1-\epsilon-\epsilon u)}}
$$

The explicit expression of this integral is

$$
\begin{aligned}
\alpha\left(\boldsymbol{e}_{1} ; \xi=1, \epsilon\right)= & \frac{\sqrt{2}}{\pi(1+4 \epsilon)\left(1-2 \epsilon+8 \epsilon^{2}\right) \sqrt{\epsilon} \sqrt{1+16 \epsilon^{2}}}\left[\pi \sqrt{1+16 \epsilon^{2}} \sqrt{2 \epsilon}\left(2 \epsilon^{2}-\epsilon+1\right)+4 \mathrm{i} \pi \epsilon(4 \epsilon-1)\right. \\
& +\operatorname{Arcsin}(4 \epsilon-1) \sqrt{1+16 \epsilon^{2}} 2 \sqrt{2} \epsilon^{3 / 2}(4 \epsilon-1)+2 \epsilon(1-4 \epsilon) \ln \epsilon+4 \epsilon(1-4 \epsilon) \ln (1-4 \epsilon) \\
& \left.+2 \epsilon(4 \epsilon-1) \ln \left(-16 \epsilon^{3}+\sqrt{1-2 \epsilon} \sqrt{16 \epsilon^{2}+1}+8 \epsilon^{2}-\epsilon+1\right)\right] .
\end{aligned}
$$

Its expansion for $\epsilon \rightarrow 0$ leads to:

$$
\alpha\left(\boldsymbol{e}_{1} ; \xi=1, \epsilon\right) \underset{\epsilon \rightarrow 0}{=} 2+\frac{2 \sqrt{2}}{\pi} \sqrt{\epsilon} \ln \epsilon+\cdots
$$


c. Computation of $\widehat{P}(\mathbf{0} \mid \mathbf{0} ; \xi, \epsilon) \quad$ Using the definition of $P_{b}$ from Eq. (96), the propagator $\widehat{P}(\mathbf{0} \mid \mathbf{0} ; \xi, \epsilon)$ can be written

$$
\widehat{P}(\mathbf{0} \mid \mathbf{0} ; \xi, \epsilon)=\int_{0}^{2 \pi} \frac{\mathrm{d} k_{x}}{2 \pi} P_{b}\left(k_{x} ; \xi\right)
$$

where $P_{b}\left(k_{x} ; \xi\right)$ is defined by Eqs. (102) to (107). We then express $\widehat{P}(\mathbf{0} \mid \mathbf{0} ; \xi, \epsilon)$ as the following integral

$$
\widehat{P}(\mathbf{0} \mid \mathbf{0} ; \xi, \epsilon)=\int_{0}^{2 \pi} \frac{\mathrm{d} k_{x}}{2 \pi} \frac{2(1-2 \epsilon)}{1-\xi \cos k_{x}+(1-4 \epsilon) \sqrt{\left(1-2 \xi \epsilon \cos k_{x}\right)^{2}-\xi^{2}(1-2 \epsilon)^{2}}} .
$$

With the change of variable $u=\cos k_{x}$, this integral is rewritten as

$$
\widehat{P}(\mathbf{0} \mid \mathbf{0} ; \xi, \epsilon)=\frac{2}{\pi} \int_{0}^{\pi} \frac{\mathrm{d} u}{\sqrt{1-u^{2}}} \frac{1-2 \epsilon}{1-\xi u+(1-4 \epsilon) \sqrt{(1-2 \xi \epsilon u)^{2}-\xi^{2}(1-2 \epsilon)^{2}}} .
$$

In the limit where $\xi \rightarrow 1$ and $\epsilon \rightarrow 0$ this quantity diverges. Studying the integrand, we show that this divergence is located near $u=1$. Introducing a new integration variable $v=1-u$, we obtain that, for $\xi \rightarrow 1$, the integral is estimated at leading order by

$$
\widehat{P}(\mathbf{0} \mid \mathbf{0} ; \xi, \epsilon) \underset{\xi \rightarrow 1}{\sim} \frac{1-2 \epsilon}{\pi \epsilon(1-4 \epsilon)} \int_{0}^{2} \frac{\mathrm{d} v}{\sqrt{v} \sqrt{2-v} \sqrt{v+\frac{1-\xi}{2 \epsilon} \sqrt{v+\frac{1}{\epsilon}-2}}} .
$$

The integral over $v$ is conveniently expressed in terms of the complete elliptic integral of the first kind $\mathrm{K}(x)$ :

$$
\widehat{P}(\mathbf{0} \mid \mathbf{0} ; \xi, \epsilon) \underset{\xi \rightarrow 1}{\sim} \frac{1-2 \epsilon}{\pi \epsilon(1-4 \epsilon)} \frac{2 \sqrt{2} \epsilon}{\sqrt{1-\xi}} \mathrm{K}\left(\frac{2 \epsilon(4 \epsilon-1-\xi)}{1-\xi}\right) .
$$

We use the following useful expansion of $\mathrm{K}(x)$ :

$$
\mathrm{K}\left(-\frac{1}{x}\right) \underset{x \rightarrow 0^{+}}{\sim} \frac{\sqrt{x}}{2} \ln \frac{1}{x},
$$

to finally obtain the expansion of $\widehat{P}(\mathbf{0} \mid \mathbf{0} ; \xi, \epsilon)$ at leading order in $(1-\xi)$ :

$$
\widehat{P}(\mathbf{0} \mid \mathbf{0} ; \xi, \epsilon) \underset{\xi \rightarrow 1}{\sim} \frac{\sqrt{1-2 \epsilon}}{\pi \sqrt{2 \epsilon}(1-4 \epsilon)} \ln \frac{1}{1-\xi} .
$$

Finally, recalling the expressions of $\widehat{F}^{*}\left(\mathbf{0}\left|\boldsymbol{e}_{1}\right| \boldsymbol{e}_{ \pm 1} ; \xi, \epsilon\right)$ in terms of the functions $\alpha$ and of the propagator $\widehat{P}(\mathbf{0} \mid \mathbf{0} ; \xi, \epsilon)$ (Eqs. (91) and (92)), we use the results from Eqs. (121), (116) and (128), we obtain the following expansions

$$
\widehat{F}^{*}\left(\mathbf{0}\left|\boldsymbol{e}_{1}\right| \boldsymbol{e}_{ \pm 1} ; \xi, \epsilon\right) \underset{\xi \rightarrow 1}{=} A_{ \pm 1}(\epsilon)+C(\epsilon) \frac{1}{\ln \frac{1}{1-\xi}}+\ldots,
$$

where $A_{ \pm 1}(\epsilon)$ and $C(\epsilon)$ have the following expansions when $\epsilon \rightarrow 0$ :

$$
\begin{gathered}
A_{1}(\epsilon) \underset{\epsilon \rightarrow 0}{\sim} 1 \\
A_{-1}(\epsilon) \underset{\epsilon \rightarrow 0}{\sim} \frac{\sqrt{2}}{\pi} \sqrt{\epsilon} \ln \frac{1}{\epsilon} \\
C(\epsilon) \underset{\epsilon \rightarrow 0}{\sim}-\pi \sqrt{\epsilon} .
\end{gathered}
$$

Recalling that the additional jump probability $\epsilon=D\left(\rho_{0}\right)$ is a vanishing function of $\rho$ when $r h o_{0} \rightarrow 0$, the conditional first-passage densities can be rewritten in terms of $\rho_{0}$ as follows:

$$
\widehat{F}^{*}\left(\mathbf{0}\left|\boldsymbol{e}_{1}\right| \boldsymbol{e}_{ \pm 1} ; \xi, \rho_{0}\right) \underset{\xi \rightarrow 1}{=} A_{ \pm 1}\left(\rho_{0}\right)+C\left(\rho_{0}\right) \frac{1}{\ln \frac{1}{1-\xi}}+\ldots
$$

where $A_{ \pm 1}\left(\rho_{0}\right)$ and $C\left(\rho_{0}\right)$ have the following expansions when $\rho_{0} \rightarrow 0$ :

$$
\begin{aligned}
& A_{1}\left(\rho_{0}\right) \underset{\epsilon \rightarrow 0}{\sim} 1 \\
& A_{-1}\left(\rho_{0}\right) \underset{\epsilon \rightarrow 0}{\propto} \frac{\sqrt{2}}{\pi} \sqrt{D\left(\rho_{0}\right)} \ln \frac{1}{D\left(\rho_{0}\right)} \\
& C\left(\rho_{0}\right) \underset{\epsilon \rightarrow 0}{\propto}-\pi \sqrt{D\left(\rho_{0}\right)} .
\end{aligned}
$$




\section{Evaluation of $\Sigma(\xi, \epsilon)$}

The evaluation of the sum $\Sigma(\xi, \epsilon)$ is conveniently done by adopting a continuous-space description of the system. Following the approach proposed by Arkhincheev and Baskin $[5,44]$, we define by $G\left(x, y \mid x_{0}, y_{0} ; t\right)$ the propagator associated with the random walk of a vacancy starting from site $\left(x_{0}, y_{0}\right)$ and arriving at $(x, y)$. The diffusion equation verified by $G$ is then

$$
\left\{\begin{array}{l}
\frac{\partial G}{\partial t}=\left[D_{1} \delta(y)+D\left(\rho_{0}\right)\right] \frac{\partial^{2} G}{\partial x^{2}}+D_{2} \frac{\partial^{2} G}{\partial y^{2}} \\
G\left(x, y \mid x_{0}, y_{0} ; t=0\right)=\delta\left(x-x_{0}\right) \delta\left(y-y_{0}\right)
\end{array}\right.
$$

where $D_{1}$ (resp. $\left.D_{2}\right)$ is the diffusion coefficient of the vacancy in the $x$ (resp. $y$ ) direction, and $D\left(\rho_{0}\right)$ is the additional jump probability experienced by the vacancy due to the effective diffusion of the TP. This coefficient is not explicitly known, and is only assumed to vanish in the limit $\rho_{0} \rightarrow 0$.

We now solve the diffusion equation (137) in order to obtain an expression for $G\left(x, y \mid x_{0}, y_{0} ; t\right)$. We introduce the continuous Laplace transform, defined for any time-dependent function by

$$
\widehat{f}(s)=\int_{0}^{\infty} \mathrm{d} t \mathrm{e}^{-s t} f(t)
$$

and the Fourier transform along the $x$-direction, defined for any $x$-dependent function:

$$
\widetilde{g}(k)=\int_{-\infty}^{\infty} \mathrm{d} x \mathrm{e}^{-\mathrm{i} k x} g(x) .
$$

The Fourier-Laplace transform of (137) is

$$
\left[s+D\left(\rho_{0}\right) k^{2}-D_{2} \frac{\partial^{2}}{\partial y^{2}}\right] \widehat{\widetilde{G}}\left(k, y \mid x_{0}, y_{0} ; s\right)=\mathrm{e}^{-\mathrm{i} k x_{0}} \delta\left(y-y_{0}\right)
$$

We first assume that $y_{0}>0$. On each of the three domains $]-\infty, 0[] 0,, y_{0}[$ and $] y_{0}, \infty[$, Eq. (140) becomes

$$
\left[s+D\left(\rho_{0}\right) k^{2}-D_{2} \frac{\partial^{2}}{\partial y^{2}}\right] \widehat{\widetilde{G}}\left(k, y \mid x_{0}, y_{0} ; s\right)=0
$$

whose general solution is

$$
\widehat{\widetilde{G}}\left(k, y \mid x_{0}, y_{0} ; s\right)=A \exp \left(-\sqrt{\frac{s+D\left(\rho_{0}\right) k^{2}}{D_{2}}}\right)+B \exp \left(\sqrt{\frac{s+D\left(\rho_{0}\right) k^{2}}{D_{2}}}\right),
$$

where $A$ and $B$ are two constants to be determined. The physically relevant solutions on the three domains take the form

$$
\widehat{\widetilde{G}}\left(k, y \mid x_{0}, y_{0} ; s\right)= \begin{cases}B_{1} \exp \left(\sqrt{\frac{s+D\left(\rho_{0}\right) k^{2}}{D_{2}}} y\right) & \text { if } y<0 \\ A_{2} \exp \left(-\sqrt{\frac{s+D\left(\rho_{0}\right) k^{2}}{D_{2}}} y\right)+B_{2} \exp \left(\sqrt{\frac{s+D\left(\rho_{0}\right) k^{2}}{D_{2}}} y\right) & \text { if } 0<y<y_{0}, \\ A_{3} \exp \left(-\sqrt{\frac{s+D\left(\rho_{0}\right) k^{2}}{D_{2}}} y\right) & \text { if } y_{0}<y\end{cases}
$$

There are four constants $\left(B_{1}, A_{2}, B_{2}\right.$ and $\left.A_{3}\right)$ to be determined. The continuity of $G$ as a function of $y$ yields the following relation:

$$
\left\{\begin{array}{l}
B_{1}=A_{2}+B_{2} \\
A_{3}=A_{2}+B_{2} \exp \left(2 \sqrt{\frac{s+D\left(\rho_{0}\right) k^{2}}{D_{2}}} y\right)
\end{array}\right.
$$


so that we write

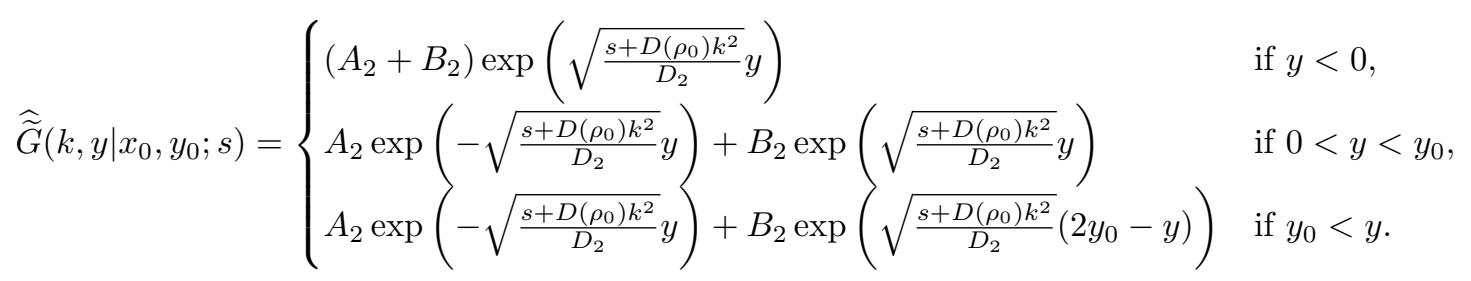

The constants $A_{2}$ and $B_{2}$ are obtained by integrating Eq. (141) respectively over the intervals $[-\eta, \eta]$ and $\left[y_{0}-\eta, y_{0}+\eta\right]$ and by taking the limits $\eta \rightarrow 0$. On the intervals $]-\infty, 0[$ and $] 0, y_{0}[$, the following relation holds

$$
\widehat{\widetilde{G}}\left(k, y \mid x_{0}, y_{0} ; s\right)=A_{2} \exp \left(-\sqrt{\frac{s+D\left(\rho_{0}\right) k^{2}}{D_{2}}}|y|\right)+B_{2} \exp \left(\sqrt{\frac{s+D\left(\rho_{0}\right) k^{2}}{D_{2}}} y\right) .
$$

We integrate Eq. (141) over $[-\eta, \eta]$ and using the following general relation (which holds for any $\alpha>0$ )

$$
\int_{-\eta}^{\eta} \mathrm{d} y \frac{\partial^{2}}{\partial y^{2}}\left[\mathrm{e}^{-\alpha|y|}\right]=\int_{-\eta}^{\eta} \mathrm{d} y \alpha[\alpha-2 \delta(y)] \mathrm{e}^{-\alpha|y|} \underset{\eta \rightarrow 0}{\rightarrow}-2 \alpha
$$

we obtain the relation

$$
D_{1} k^{2}\left(A_{2}+B_{2}\right)+2 A_{2} \sqrt{D_{2}\left[s+D\left(\rho_{0}\right) k^{2}\right]}=0 .
$$

On the intervals $] 0, y_{0}\left[\right.$ and $\left[y_{0}, \infty[\right.$, the following expression of $\widehat{\widetilde{G}}$ holds:

$$
\widehat{\widetilde{G}}\left(k, y \mid x_{0}, y_{0} ; s\right)=A_{2} \exp \left(-\sqrt{\frac{s+D\left(\rho_{0}\right) k^{2}}{D_{2}}} y\right)+B_{2} \exp \left(-\sqrt{\frac{s+D\left(\rho_{0}\right) k^{2}}{D_{2}}} y_{0}\right) \exp \left(-\sqrt{\frac{s+D\left(\rho_{0}\right) k^{2}}{D_{2}}}\left|y-y_{0}\right|\right) .
$$

We integrate Eq. (141) over $\left[y_{0}-\eta, y_{0}+\eta\right]$, and using again Eq. (147), we obtain the expression of $B_{2}$ :

$$
B_{2}=\frac{1}{2 \sqrt{D_{2}\left[s+D\left(\rho_{0}\right) k^{2}\right]}} \exp \left(-\mathrm{i} k x_{0}-\sqrt{\frac{s+D\left(\rho_{0}\right) k^{2}}{D_{2}}} y_{0}\right) .
$$

The relation between $A_{2}$ and $B_{2}$ yields

$$
A_{2}=-\frac{D_{1} k^{2}}{D_{1} k^{2}+2 \sqrt{D_{2}\left[s+D\left(\rho_{0}\right) k^{2}\right]}} \frac{1}{2 \sqrt{D_{2}\left[s+D\left(\rho_{0}\right) k^{2}\right]}} \exp \left(-\mathrm{i} k x_{0}-\sqrt{\frac{s+D\left(\rho_{0}\right) k^{2}}{D_{2}}} y_{0}\right) .
$$

In what follows, we will only consider the propagator of a random walk starting from a generic point $\left(x_{0}, y_{0}\right)$ and arriving at the origin $(0,0)$, so that we will take $y=0$. Generalizing the calculation to the case of a starting point located in the domain $y_{0}<0$, we finally obtain the general expression

$$
\widehat{\widetilde{G}}\left(k, 0 \mid x_{0}, y_{0} ; s\right)=\frac{\exp \left(-\mathrm{i} k x_{0}-\sqrt{\frac{s+D\left(\rho_{0}\right) k^{2}}{D_{2}}}\left|y_{0}\right|\right)}{D_{1} k^{2}+2 \sqrt{D_{2}\left[s+D\left(\rho_{0}\right) k^{2}\right]}} .
$$

We now study the asymptotic behavior of the sum $\Sigma(\xi, \epsilon)=\sum_{\boldsymbol{Z} \neq \mathbf{0}} \widehat{F}^{*}\left(\mathbf{0}\left|\boldsymbol{e}_{1}\right| \boldsymbol{Z} ; \xi, \epsilon\right)$ in the limit where $\xi \rightarrow 1$ (long-time limit). In the continuous-space and time description, we replace the sum by the following integral

$$
\Sigma\left(s, \rho_{0}\right)=\int \mathrm{d} x_{0} \int \mathrm{d} y_{0} \widehat{F}\left(0,0 \mid x_{0}, y_{0} ; s\right) .
$$

Writing the following renewal equation

$$
\widehat{F}\left(0,0 \mid x_{0}, y_{0} ; s\right)=\frac{\widehat{G}\left(0,0 \mid x_{0}, y_{0} ; s\right)}{\widehat{G}(0,0 \mid 0,0 ; s)},
$$


we get

$$
\Sigma\left(s, \rho_{0}\right)=\frac{1}{\widehat{G}(0,0 \mid 0,0 ; s)} \int \mathrm{d} x_{0} \int \mathrm{d} y_{0} \widehat{G}\left(0,0 \mid x_{0}, y_{0} ; s\right) .
$$

Taking the inverse Fourier transform of Eq. (152), we write

$$
\widehat{G}\left(x, 0 \mid x_{0}, y_{0} ; s\right)=\int_{-\infty}^{\infty} \frac{\mathrm{d} k}{2 \pi} \exp (\mathrm{i} k x) \frac{\exp \left(-\mathrm{i} k x_{0}-\sqrt{\frac{s+D\left(\rho_{0}\right) k^{2}}{D_{2}}}\left|y_{0}\right|\right)}{D_{1} k^{2}+2 \sqrt{D_{2}\left[s+D\left(\rho_{0}\right) k^{2}\right]}} .
$$

We now evaluate separately $\widehat{G}(0,0 \mid 0,0 ; s)$ and $\int \mathrm{d} x_{0} \mathrm{~d} y_{0} \widehat{G}\left(0,0 \mid x_{0}, y_{0} ; s\right)$ :

- using Eq. (156), we obtain the expression of $\widehat{G}(0,0 \mid 0,0 ; s)$ :

$$
\begin{aligned}
\widehat{G}(0,0 \mid 0,0 ; s) & =\int_{-\infty}^{\infty} \frac{\mathrm{d} k}{2 \pi} \frac{1}{D_{1} k^{2}+2 \sqrt{D_{2}\left[s+D\left(\rho_{0}\right) k^{2}\right]}}, \\
& =\frac{1}{\pi} \int_{0}^{\infty} \mathrm{d} k \frac{1}{D_{1} k^{2}+2 \sqrt{D_{2}\left[s+D\left(\rho_{0}\right) k^{2}\right]}} .
\end{aligned}
$$

In the long-time limit $(s \rightarrow 0)$, the integral is dominated by the small values of the variable $k$. Introducing a cutoff value $A$, we write

$$
\widehat{G}(0,0 \mid 0,0 ; s) \sim \frac{1}{\pi} \int_{0}^{A} \mathrm{~d} k \frac{1}{2 \sqrt{D_{2}\left[s+D\left(\rho_{0}\right) k^{2}\right]}} .
$$

Using the following integral (for $a>0$ ),

$$
\int_{0}^{A} \mathrm{~d} x \frac{1}{\sqrt{a^{2}+x^{2}}}=\ln \left(\frac{A}{a}+\sqrt{\frac{A}{a}+1}\right),
$$

we obtain the leading order behavior of $\widehat{G}(0,0 \mid 0,0 ; s)$ when $s \rightarrow 0$ :

$$
\widehat{G}(0,0 \mid 0,0 ; s) \underset{s \rightarrow 0}{\sim} \frac{1}{4 \pi \sqrt{D_{2}}} \frac{1}{\sqrt{D\left(\rho_{0}\right)}} \ln \frac{1}{s} .
$$

- the integral $\int \mathrm{d} x_{0} \int \mathrm{d} y_{0} \widehat{G}\left(0,0 \mid x_{0}, y_{0} ; s\right)$, using Eq. (156), yields:

$$
\begin{aligned}
\int \mathrm{d} x_{0} \int \mathrm{d} y_{0} \widehat{G}\left(0,0 \mid x_{0}, y_{0} ; s\right) & =\int \mathrm{d} x_{0} \int \mathrm{d} y_{0} \int_{-\infty}^{\infty} \frac{\mathrm{d} k}{2 \pi} \frac{\exp \left(-\mathrm{i} k x_{0}-\sqrt{\frac{s+D\left(\rho_{0}\right) k^{2}}{D_{2}}}\left|y_{0}\right|\right)}{D_{1} k^{2}+2 \sqrt{D_{2}\left[s+D\left(\rho_{0}\right) k^{2}\right]}} \\
& =\int_{-\infty}^{\infty} \frac{\mathrm{d} k}{\pi} \int \mathrm{d} x_{0} \frac{\exp \left(-\mathrm{i} k x_{0}\right)}{D_{1} k^{2}+2 \sqrt{D_{2}\left[s+D\left(\rho_{0}\right) k^{2}\right]}} \sqrt{\frac{D_{2}}{s+D\left(\rho_{0}\right) k^{2}}} \\
& =\int_{-\infty}^{\infty} \mathrm{d} k \frac{2 \delta(k)}{\frac{D_{2}}{D_{1} k^{2}+2 \sqrt{D_{2}\left[s+D\left(\rho_{0}\right) k^{2}\right]}}} \\
& =\frac{1}{s}
\end{aligned}
$$

Finally, using the estimates from Eqs. (161) and (165) in Eq. (155), we get

$$
\Sigma\left(s, \rho_{0}\right) \underset{s \rightarrow 0}{\sim} \frac{4 \pi \sqrt{D_{2}} \sqrt{D\left(\rho_{0}\right)}}{s \ln (1 / s)} .
$$

In the long-time limit, we get the equivalent discrete time description in terms of the variable $\xi$ :

$$
\Sigma\left(\xi, \rho_{0}\right) \underset{\xi \rightarrow 1}{\sim} \frac{4 \pi \sqrt{D_{2}} \sqrt{D\left(\rho_{0}\right)}}{(1-\xi) \ln \frac{1}{1-\xi}} .
$$




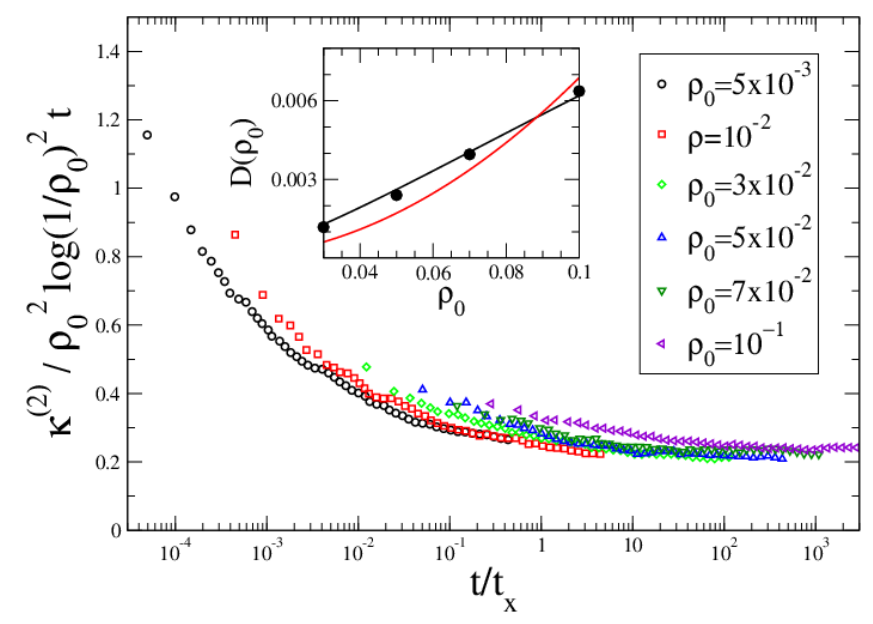

FIG. 7. (Color online) Variance of the TP when its motion is constrained on the backbone, rescaled by the time $t$ and $\rho_{0}^{2} \log \left(1 / \rho_{0}\right)^{2}$, according to Eq. (11) of the main text, for several values of $\rho_{0}$. Inset: the black dots represent the diffusion coefficient $D\left(\rho_{0}\right)=\lim _{t \rightarrow \infty} \kappa^{(2)}(t) / 2 t$ measured in simulations and the black line is the best fit obtained with the function $f(x)=a x^{2} \log (1 / x)^{2} \quad(\mathrm{a}=0.2336)$. For comparison we also show the best fit obtained with the function $g(x)=b x^{2}$, with $b=1.375$ (red line).

\section{Expression of the variance}

We recall the expression of the generating function associated with the variance of the TP position:

$$
\widehat{\kappa}^{(2)}\left(\xi, \rho_{0}\right)=-2 \rho_{0} \Sigma\left(\xi, \rho_{0}\right) \frac{\widehat{F}_{1}^{*}-\widehat{F}_{-1}^{*}-1}{\left(\widehat{F}_{1}^{*}-1+\widehat{F}_{-1}^{*}\right)\left(\widehat{F}_{1}^{*}+1-\widehat{F}_{-1}^{*}\right)} .
$$

Using the results from Eqs. (167) and (133), we obtain the expansion of the generating function associated with the variance up to a numerical prefactor, where we first take the long-time limit $(\xi \rightarrow 1)$ and ultimately the high-density limit $\left(\rho_{0} \rightarrow 0\right)$ :

$$
\widehat{\kappa}^{(2)}\left(\xi, \rho_{0}\right) \propto \frac{\rho_{0}}{(1-\xi)^{2}} \sqrt{D\left(\rho_{0}\right)} \ln \frac{1}{D\left(\rho_{0}\right)} .
$$

Using a Tauberian theorem, we retrieve the time-dependence of the cumulant and the expression presented in the main text:

$$
\lim _{t \rightarrow \infty} \frac{\kappa^{(2)}(t)}{t} \underset{\rho_{0} \rightarrow 0}{\propto} \rho_{0} \sqrt{D\left(\rho_{0}\right)} \ln \frac{1}{D\left(\rho_{0}\right)} .
$$

From this expression we obtain Eq. (10) of the main text, which turns to be in good agreement with numerical simulations, as shown in Fig. 7.

\section{NUMERICAL SIMULATIONS}

For the case where the TP is constrained on the backbone, the data reported are obtained via Monte Carlo numerical simulations of vacancy dynamics. We considered a lattice of size $L_{x} \times L_{y}=1000^{2}$ with periodic boundary conditions and a number of vacancies $M=\rho_{0} L_{x} L_{y}$. At each Monte Carlo step, the position of all vacancies is updated according to the evolution rules described in Section I. If a vacancy exchanges its position with the TP, the TP position is also updated and the displacement is measured. Reported data are averaged over some thousands of realizations.

In the case where the TP is allowed to visit the teeth of the lattice, for values of $\rho_{0} \leq 10^{-3}$ the reported data are obtained via Monte Carlo simulations of vacancy dynamics, with the same lattice size as above. For larger values of $\rho_{0}$ we performed Monte Carlo simulations of particle dynamics. At each Monte Carlo step, all particles (included the TP) attempt a move on a neighbor site with the probabilities given in the main text, and the move is accepted 
if the target site is empty. In this case, the lattice size is $L_{x} \times L_{y}=200^{2}$ with periodic boundary conditions and the number of particles is $N=\left(1-\rho_{0}\right) L_{x} L_{y}$. 